\title{
2018 Atmospheric Motion Vector (AMV) Intercomparison Study
}

\author{
David Santek $^{1, *}$, Richard Dworak ${ }^{1}$, Sharon Nebuda ${ }^{1}$, Steve Wanzong ${ }^{1}\left(\mathbb{D}\right.$, Régis Borde $^{2}$, \\ Iliana Genkova ${ }^{3}$, Javier García-Pereda ${ }^{4}$, Renato Galante Negri ${ }^{5}$, Manuel Carranza ${ }^{6}$, \\ Kenichi Nonaka ${ }^{7}$, Kazuki Shimoji ${ }^{7}$, Soo Min Oh ${ }^{8,9}{ }^{(D}$, Byung-Il Lee ${ }^{9}$, Sung-Rae Chung ${ }^{9}$, \\ Jaime Daniels ${ }^{10}$ and Wayne Bresky ${ }^{11}$
}

1 Space Science and Engineering Center (SSEC), University of Wisconsin-Madison, 1225 West Dayton Street, Madison, WI 53706, USA

2 EUMETSAT, Eumetsat Allee 1, 64295 Darmstadt, Germany

3 I.M. Systems Group at National Centers for Environmental Prediction (NCEP), National Oceanic and Atmospheric Administration (NOAA), 5830 University Research Court, College Park, MD 20740, USA

4 Satellite Application Facility on support to Nowcasting and Very short range forecasting (NWCSAF), Agencia Estatal de Meteorología (AEMET), Leonardo Prieto Castro 8, 28040 Madrid, Spain

5 Center for Weather Prediction and Climate Studies (CPTEC), National Institute for Space Research (INPE), Cachoeira Paulista, São Paulo 12630-000, Brazil

GMV INSYEN at EUMETSAT, Eumetsat Allee 1, 64295 Darmstadt, Germany

7 Japan Meteorological Agency, 1-3-4 Otemachi, Chiyoda-ku, Tokyo 100-8122, Japan

8 Department of Physics and Astronomy, Seoul National University, 1, Gwanak-ro, Gwanak-gu, Seoul 08826, Korea

9 National Meteorological Satellite Center (NMSC), Korea Meteorological Administration (KMA), 64-18, Guam-gil, Gwanghyewon-myeon, Jincheon-gun, Chungcheongbuk-do 27803, Korea

10 Center for Satellite Applications and Research (STAR), National Environmental Satellite, Data and Information Service (NESDIS), National Oceanic and Atmospheric Administration (NOAA), 5830 University Research Court, College Park, MD 20740, USA

11 I.M. Systems Group at Center for Satellite Applications and Research (STAR), National Environmental Satellite, Data and Information Service (NESDIS), National Oceanic and Atmospheric Administration (NOAA), 5830 University Research Court, College Park, MD 20740, USA

* Correspondence: dave.santek@ssec.wisc.edu

Received: 31 July 2019; Accepted: 20 September 2019; Published: 26 September 2019

\begin{abstract}
Atmospheric Motion Vectors (AMVs) calculated by six different institutions (Brazil Center for Weather Prediction and Climate Studies/CPTEC/INPE, European Organization for the Exploitation of Meteorological Satellites/EUMETSAT, Japan Meteorological Agency/JMA, Korea Meteorological Administration/KMA, Unites States National Oceanic and Atmospheric Administration/NOAA, and the Satellite Application Facility on Support to Nowcasting and Very short range forecasting/NWCSAF) with JMA's Himawari-8 satellite data and other common input data are here compared. The comparison is based on two different AMV input datasets, calculated with two different image triplets for 21 July 2016, and the use of a prescribed and a specific configuration. The main results of the study are summarized as follows: (1) the differences in the AMV datasets depend very much on the 'AMV height assignment' used and much less on the use of a prescribed or specific configuration; (2) the use of the 'Common Quality Indicator (CQI)' has a quantified skill in filtering collocated AMVs for an improved statistical agreement between centers; (3) Among the six AMV operational algorithms verified by this AMV Intercomparison, JMA AMV algorithm has the best overall performance considering all validation metrics, mainly due to its new height assignment method: 'Optimal estimation method considering the observed infrared radiances, the vertical profile of the Numerical Weather Prediction wind, and the estimated brightness temperature using a radiative transfer model'.
\end{abstract}


Keywords: Atmospheric Motion Vectors (AMVs); Intercomparison; Himawari; CPTEC/INPE; EUMETSAT; JMA; KMA; NOAA; NWCSAF

\section{Introduction}

The use of satellite-derived cloud displacements to infer atmospheric motion (AMVs or Atmospheric Motion Vectors) has been investigated since the first weather satellites were launched. In the early 1960s, Tetsuya Fujita developed analysis techniques to use cloud pictures from the first Television Infrared Observation Satellite (TIROS), a polar orbiter, for estimating the velocity of tropospheric winds [1]. Throughout the 1970s and early 1980s, AMVs were produced from geostationary satellite data using a combination of automated and manual techniques.

The derivation of Atmospheric Motion Vectors (AMVs) has traditionally been based on the following steps:

- The reading and preprocessing of the satellite data, including, for example, the normalization of satellite visible images.

- The location of suitable 'tracers' in an initial image: cloud features, and humidity features in cloudless areas in water vapor images.

- The location of those tracers in a later image: tracking process. The features can change their shape or even disappear, but enough of them survive to produce a significant number of AMVs; with short time intervals, up to $15 \mathrm{~min}$, the evolution is smaller and more vectors can be calculated.

- The 'height assignment' of the tracers: the pressure level of the feature must be determined to locate the AMVs in a tridimensional position in the atmosphere.

- The calculation of the AMV vectors, considering the geographical displacement of the tracers between the initial image and the later image.

- A quality control process, so that only the AMVs with better quality are accepted.

The following is a brief chronology on the generation of geostationary AMVs from the primary producers:

- In 1969, the NOAA (Unites States National Oceanic and Atmospheric Administration) National Environmental Satellite Service (NESS, now NESDIS) began routine production of a completely manual technique for producing wind vectors from viewing time loops of visible satellite images, resulting in approximately 300 AMVs per day [2]. The technique was partially computerized in the 1970s, automated in the 1990s, and was recently updated for the GOES-R series satellites [3].

- The University of Wisconsin-Madison (UW) Space Science and Engineering Center (SSEC) developed a computerized algorithm to manually track clouds from the imagery of the first generation of geostationary weather satellites [4]. This technique was fully automated in the 1990s [5], and variations of this technique are still in use today.

- The extraction of AMVs from Meteosat infrared imagery has been operational at the European Space Operations Center (ESOC) and European Organization for the Exploitation of Meteorological Satellites (EUMETSAT) since the late 1970s. After several major improvements, the extraction technique reached a stage in 1991, where the majority of the vectors represented the local wind field [6]. The algorithm was updated in 2012, including the 'Cross-correlation contribution (CCC) method' to set the AMV altitude [7].

- GMS satellite was launched in July 1977 as the first Japanese geostationary meteorological satellite and operated from 1978 to 1984. The Japan Meteorological Agency/Meteorological Satellite Center (JMA/MSC) routinely began AMV derivation provided twice a day from the GMS satellite imagery in 1978, by using the Cloud Wind Estimation System (CWES) [8]. The derivation system was fully automated in 2003, and AMVs are today calculated every hour by the latest algorithm adapted to Himawari-8. 
- Atmospheric Motion Vectors have been produced operationally at Brazil Center for Weather Prediction and Climate Studies (CPTEC/INPE) since 1998. The original algorithm was based on the routines developed by ESOC and adapted to use GOES-8 $10.8 \mu \mathrm{m}$ infrared images [9]. AMVs from the water vapor channel were added to the operational suite, followed by the $3.9 \mu \mathrm{m}$ and visible channels in 2006 to estimate winds from the lower troposphere only [10]. More recently, the algorithm has been adapted to data from GOES-16.

- Korea Meteorological Administration (KMA) developed an AMV algorithm for COMS satellite in 2003. Now it is in charge of the AMV algorithm for GEO-KOMPSAT-2 satellites [11].

- The NWCSAF (Satellite Application Facility on Support to Nowcasting and Very short range forecasting) delivered the first version of its AMV product "“HRW/High Resolution Winds" for MSG satellites, inside the 2004 release of its software package "NWC/MSG". A major change occurred in 2011, with the inclusion of 'CCC method' for the AMV height assignment. Progressive extensions have permitted to calculate AMVs with additional geostationary satellite series throughout the world (GOES-N in 2016, Himawari-8/9 in 2018, and GOES-R in 2019) [12].

However, it was not until 2008 that a coordinated effort was organized to compare the AMVs from the primary operational AMV producing centers. Overall, this resulted in a total of three 'Atmospheric Motion Vector (AMV) Intercomparison studies', which took place over the last decade: Genkova et al. 2008 and 2010 [13,14], and Santek et al. 2014 [15].

The studies assessed how satellite-derived cloudy AMVs compared in terms of coverage, speed, direction, and cloud height.

The algorithms used for the AMV calculation, and the geostationary satellites with which this process is done, have evolved very much since then, with a new generation of satellites with additional spectral channels bearing new information on cloud microphysics, and with a higher temporal resolution which allows a better understanding of the characteristics of the tracked features.

All this has defined the need for a new 'AMV Intercomparison study' in 2018, and the need to formally publish for the first time the results of these Intercomparisons. The study would act as an input for the development of all AMV algorithms, for their optimal use with this new generation of geostationary satellites.

Three main goals have been considered for this study:

(1) To verify the advantages of the calculation of AMVs with the new generation of geostationary satellites that began with Himawari-8, with better spatial and temporal resolution and new spectral channels, with respect to those calculated with previous satellite series like MSG.

(2) To compute a 'Common Quality Indicator (CQI)' for all AMV algorithms, using the self-contained Fortran module defined by EUMETSAT and NOAA/NESDIS and distributed to all AMV producers by the International Winds Working Group (IWWG) in May 2017, to verify if there is a better agreement between the different AMV datasets using this CQI.

(3) To extract conclusions about the best options for the calculation of AMVs with this new generation of geostationary satellites, taking into account the options defined by the different centers for their AMV calculation.

\section{Materials and Methods}

The geostationary AMV algorithms defined by the following six AMV producers have been analyzed here. The three-letter abbreviations have been used as identifiers of the AMV datasets throughout the remainder of this article:

- BRZ: Brazil Center for Weather Prediction and Climate Studies (CPTEC/INPE)

- EUM: European Organization for the Exploitation of Meteorological Satellites (EUMETSAT)

- JMA: Japan Meteorological Agency

- KMA: Korea Meteorological Administration 
- $\quad$ NOA: United States National Oceanic and Atmospheric Administration (NOAA)

- NWC: Satellite Application Facility on Support to Nowcasting (NWCSAF)

Full information on the AMV algorithms used here can be obtained from the "Operational AMV Product Survey", available at the "International Winds Working Group (IWWG)" webpage [16]. Enumerating a summary of these characteristics:

- All algorithms are operational at the corresponding centers, although some of them not with Himawari satellites (BRZ, EUM, and NOA).

- Most products are distributed through the meteorological Global Telecommunications System (GTS), while some are produced locally (NWC).

- Many output formats are available, although all AMV algorithms include the heritage Binary Universal Form for the Representation of meteorological data (BUFR) sequence, and most of them have plans of implementing the 2018 new BUFR sequence (3.10.077) for AMVs.

- There is an important variability in the way Numerical Weather Prediction (NWP) data and other input products are used by the different AMV algorithms, and also in the way the AMV features are defined.

- Tracers based on square features of different sizes ('target sizes') between $7 \times 7$ pixels and $31 \times 31$ pixels are used for the processing by the different AMV algorithms. The square features are located in a regular grid with nominal spacings between $12 \mathrm{~km}$ and $72 \mathrm{~km}$ at subsatellite point for the different AMV algorithms, although some methods infer corrections to optimize the location of each feature.

- 'Cross-correlation method' is used for the AMV tracking by all centers except NOA, which uses the 'Sum of Squared Differences'. The 'Cross-correlation threshold' and the way the tracking is implemented is slightly different for each algorithm.

- All algorithms except BRZ calculate, for the study, the AMV displacement as the average of two intermediate components; EUM and NOA additionally use the central image of each triplet as a reference and tracks backward for the first intermediate component and then forward for the second intermediate component.

- There is an important variability in the height assignment method used by the different centers. For example, 'Cross-correlation contribution (CCC)' is used by EUM and NWC; 'Effective black body temperature (EBBT)' with optional 'Water vapor/infrared ratioing' and ' $\mathrm{CO}_{2}$ slicing' corrections are used by BRZ and KMA; an 'Optimal estimation method considering the observed infrared radiances, the vertical profile of the NWP wind and the estimated brightness temperature using a radiative transfer model' is used by JMA. Some thresholds and corrections are additionally implemented by each center for its specific implementation.

- The 'Quality Indicator without Forecast (QINF)' (based on the 'EUMETSAT Quality Indicator' [17], with a specific implementation at each center), and the 'Common Quality Indicator without Forecast (CQI)' (using a common implementation and code, such as defined previously in the Introduction) are implemented in all AMV algorithms. The 'Quality Indicator with Forecast (QIF)' (based on the 'EUMETSAT Quality Indicator' [17], with a specific implementation at each center) is implemented by all AMV algorithms except BRZ.

- The 'quality indicator threshold' and several other quality checks are different for each algorithm. Additionally, other quality methods are included by some centers: the 'Expected error' (KMA and NOA) and the 'Orographic flag' (NWC). 
The AMV outputs were originated at the different participating centers using the following input data:

- Two triplets of Himawari-8/AHI infrared $10.4 \mu \mathrm{m}$ full-disk images for 21 July 2016 at 0530-0550 UTC and 1200-1220 UTC, one of which is shown in Figure 1. Information was provided at each Himawari-8 pixel for the following variables: latitude, longitude, scanning time, satellite and solar zenith angles, radiance, and brightness temperature.

- Auxiliary files, containing land/sea mask, land cover, and elevation for each Himawari-8 pixel.

- European Centre for Medium-Range Weather Forecasts (ECMWF) ERA-Interim NWP analysis [18] for the given day, for 37 vertical levels every $6 \mathrm{~h}$, on a regular latitude/longitude grid with a resolution of 0.5 degrees. Information was provided for the following variables: geopotential, surface pressure, temperature, dew point temperature, 2-m temperature, skin temperature, relative humidity, wind components, 10-m wind components, ozone mixing ratio, total column ozone, land/sea mask, sea/ice cover, snow depth, and mean sea level pressure. Considering this, it is known that differences can exist considering different NWP analysis sources, based especially on the number of observations used in the assimilation process of each NWP model. For this study, it was assumed that the differences in the winds from the different AMV datasets are more significant than the differences caused by the use of one or other NWP model analysis in the process. This assumption is confirmed by the results, in which the differences in the mean winds of the different AMV datasets are larger than the differences in the mean winds of different NWP analysis.

- Cloud products derived by NOAA/NESDIS for the given time slots. Information was provided for the following variables: surface type, surface elevation, land class, cloud probability, cloud mask, cloud type, cloud phase, and cloud top pressure. However, due to the different cloud assignment methods and the way cloud data are used in them by the different AMV algorithms, only EUM, NOA, and NWC made actual use of these NOAA/NESDIS cloud products.

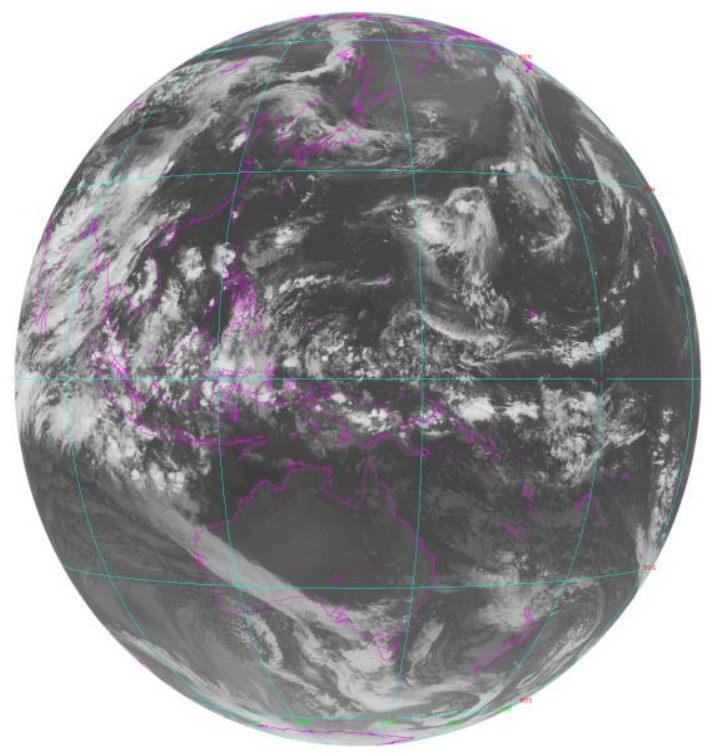

Figure 1. Himawari-8 $10.4 \mu \mathrm{m}$ satellite image for 21 July 2016 at 1200 UTC.

The Himawari-8/AHI satellite images used in the Intercomparison were equivalent to those used by the "International Cloud Working Group (ICWG)" for the "Cloud Intercomparison study", to improve synergies between both studies. The day was selected due to the variability of meteorological features found in the different regions.

The AMV outputs provided by each AMV algorithm were analyzed in three independent experiments, designed to measure differences related to specific aspects of the algorithms. Software 
tools used in the three previous Intercomparison studies (Genkova et al. 2008 and 2010 [13,14], and Santek et al. 2014 [15]) were used again, allowing for the comparison of the results in the different studies.

The AMV datasets produced by the different producers for the AMV Intercomparison, all software tools used in the AMV Intercomparison for the verification of the AMV data, and the radiosonde data used for the AMV data validation, with a text file describing how to process all these elements, have been made available inside a zipped file (File S1), such as described in the "Supplementary Materials" chapter.

The AMV output from each center for the considered experiments in this AMV Intercomparison study, included data for the following variables shown in Table 1.

Table 1. Reported variables for the AMVs (Atmospheric Motion Vectors) in all datasets.

\begin{tabular}{|c|c|c|}
\hline Parameter & Code & Description \\
\hline 1 & IDN & AMV Identification Number \\
\hline 2 & LAT[degrees] & AMV Latitude \\
\hline 3 & LON[degrees] & AMV Longitude \\
\hline 4 & TS[pixels] & Target Box Size \\
\hline 5 & SS[pixels] & Search Box Size \\
\hline 6 & $\mathrm{SPD}[\mathrm{m} / \mathrm{s}]$ & AMV Speed \\
\hline 7 & DIR[degrees] & $\begin{array}{l}\text { AMV Direction } \\
\text { (0: due North, 90: due East, 180: due South, 270: due West) }\end{array}$ \\
\hline 8 & PRES[hPa] & AMV Pressure \\
\hline 9 & $\mathrm{~L}$ & $\begin{array}{c}\text { Low-Level Correction Flag } \\
\text { (0: No correction, 1: Inversion correction, 2: Cloud base) }\end{array}$ \\
\hline 10 & NWPSPD[m/s] & Numerical Weather Prediction (NWP) Wind Speed Guess \\
\hline 11 & NWPDIR[degrees] & Numerical Weather Prediction (NWP) Wind Direction Guess \\
\hline 12 & $\mathrm{ALB}[\%]$ & $0.8 \mu \mathrm{m}$ Albedo at the Center Pixel of the Target Box \\
\hline 13 & CORR[\%] & Maximum Value of the Tracking Cross-correlation \\
\hline 14 & $\mathrm{~T}$ & $\begin{array}{c}\text { Tracking Method Flag } \\
\text { (0: Cross-correlation, 1: Sum of squared differences, 2: Other) }\end{array}$ \\
\hline 15 & PRESERR[hPa] & AMV Pressure Error \\
\hline 16 & $\mathrm{H}$ & $\begin{array}{l}\text { Height Assignment Method, as defined in page } 4 \text { of the text } \\
\text { (0: EBBT, 1: } \mathrm{CO}_{2} \text { Slicing, 2: IR/WV ratioing, 3: CCC, 4: Other) }\end{array}$ \\
\hline 17 & QINF[\%] & Quality Indicator without Forecast \\
\hline 18 & $\mathrm{QIF}[\%]$ & Quality Indicator with Forecast \\
\hline 19 & CQI[\%] & Common Quality Indicator \\
\hline
\end{tabular}

\section{Results}

\subsection{Experiment 1}

In this case, AMV producers extracted cloudy AMVs with the Himawari-8/AHI infrared $10.4 \mu \mathrm{m}$ image triplet 1200-1220 UTC, using their best options for the AMV calculation, but with prescribed target box sizes $(16 \times 16$ pixels), search scene sizes $(54 \times 54$ pixels), and target locations (with a line and column separation of 16 pixels).

This way, differences in the AMV densities and all AMV extraction processes (tracer selection, tracer tracking, height assignment, and quality control) could be compared, verifying exactly equivalent AMV datasets. 
Table 2 shows the parameter distribution for the AMV datasets, with a 'CQI threshold' of 50\% (CQI $>=50 \%$ ). This threshold is used in all AMV datasets to avoid the processing of 'low-quality AMVs'; in some elements of the Intercomparison, an additional 'CQI threshold' of $80 \%$ (CQI $>=80 \%$ ) is used to verify its impact on the improvement of the validation parameters in the different AMV datasets.

Table 2. Parameter distribution for the different AMV datasets in Experiment 1 with CQI $>=50 \%$.

\begin{tabular}{|c|c|c|c|c|c|c|}
\hline & BRZ & EUM & JMA & KMA & NOA & NWC \\
\hline Total Number of AMVs & 70,312 & 30,868 & 76,075 & 58,479 & 38,242 & 14,602 \\
\hline Common Quality Index $>=50 \%$ & 57,613 & 27,097 & 33,717 & 52,685 & 36,172 & 14,283 \\
\hline Speed $\min .(\mathrm{m} / \mathrm{s})$ & 2.56 & 2.51 & 2.50 & 2.50 & 3.00 & 2.50 \\
\hline Speed max. (m/s) & 66.05 & 139.93 & 69.97 & 97.01 & 92.29 & 68.77 \\
\hline Speed mean $(\mathrm{m} / \mathrm{s})$ & 11.81 & 13.64 & 13.29 & 13.66 & 14.06 & 13.64 \\
\hline Pressure min. (hPa) & 100.00 & 57.11 & 125.00 & 102.08 & 102.05 & 101.00 \\
\hline Pressure max. (hPa) & 1000.00 & 1004.00 & 994.32 & 997.66 & 977.82 & 965.50 \\
\hline Pressure mean $(\mathrm{hPa})$ & 533.19 & 437.37 & 496.89 & 408.80 & 432.19 & 386.40 \\
\hline Percentage of Low AMVs (\%) & 37.10 & 26.66 & 31.43 & 23.05 & 33.77 & 23.61 \\
\hline Percentage of Mid AMVs (\%) & 20.33 & 14.56 & 19.34 & 13.29 & 2.63 & 8.96 \\
\hline Percentage of High AMVs (\%) & 42.57 & 58.78 & 49.23 & 63.66 & 63.59 & 67.43 \\
\hline Speed min. of Low AMVs (m/s) & 2.56 & 2.52 & 2.50 & 2.50 & 3.00 & 2.57 \\
\hline Speed max. of Low AMVs (m/s) & 35.67 & 99.02 & 41.20 & 70.19 & 40.52 & 28.57 \\
\hline Speed mean of Low AMVs (m/s) & 9.61 & 9.33 & 9.06 & 9.67 & 10.21 & 9.95 \\
\hline Pressure min. of Low AMVs (hPa) & 700.00 & 700.23 & 700.01 & 700.00 & 700.06 & 700.00 \\
\hline Pressure max. of Low AMVs (hPa) & 1000.00 & 1004.00 & 994.32 & 997.66 & 977.82 & 965.50 \\
\hline Pressure mean of Low AMVs (hPa) & 874.49 & 823.71 & 888.89 & 820.21 & 837.59 & 782.70 \\
\hline Speed min. of Mid AMVs (m/s) & 2.61 & 2.53 & 2.50 & 2.50 & 3.06 & 2.51 \\
\hline Speed max. of Mid AMVs (m/s) & 66.05 & 94.60 & 69.25 & 70.08 & 78.86 & 48.12 \\
\hline Speed mean of Mid AMVs (m/s) & 13.61 & 14.58 & 14.03 & 14.53 & 22.14 & 17.05 \\
\hline Pressure min. of Mid AMVs (hPa) & 400.02 & 400.03 & 400.01 & 400.03 & 400.01 & 400.05 \\
\hline Pressure max. of Mid AMVs (hPa) & 699.98 & 699.77 & 699.99 & 699.90 & 449.96 & 699.50 \\
\hline Pressure mean of Mid AMVs (hPa) & 536.33 & 531.56 & 528.20 & 521.45 & 425.91 & 536.54 \\
\hline Speed min. of High AMVs (m/s) & 2.58 & 2.51 & 2.50 & 2.50 & 3.00 & 2.50 \\
\hline Speed max. of High AMVs (m/s) & 62.47 & 139.93 & 69.97 & 97.01 & 92.29 & 68.77 \\
\hline Speed mean of High AMVs (m/s) & 12.88 & 15.37 & 15.69 & 14.92 & 15.77 & 14.47 \\
\hline Pressure min. of High AMVs (hPa) & 100.00 & 57.11 & 125.00 & 102.08 & 102.05 & 101.00 \\
\hline Pressure max. of High AMVs (hPa) & 400.00 & 399.94 & 399.98 & 399.97 & 399.99 & 400.00 \\
\hline Pressure mean of High AMVs (hPa) & 234.17 & 238.80 & 234.32 & 236.29 & 217.14 & 227.70 \\
\hline
\end{tabular}

- The total number of AMVs ranges from 15,000 to 76,000, with the lower values for NWC and the larger values for JMA, and a factor of five between the numbers of AMVs of both centers. The number of AMVs is much larger with Himawari satellites than in the previous 'AMV Intercomparison' with MSG satellite [15], which found up to approximately 10,000 AMVs. This is due, in part, to the higher resolution of Himawari/AHI (2 km) versus MSG/SEVIRI $(3 \mathrm{~km})$, more oceanic regions, and fewer deserts.

- The maximum speed ranges from $66 \mathrm{~m} \mathrm{~s}^{-1}$ (BRZ) to $97 \mathrm{~m} \mathrm{~s}^{-1}$ (KMA), with outliers of $140 \mathrm{~m} \mathrm{~s}^{-1}$ for EUM.

- The minimum pressure ranges between $57 \mathrm{hPa}$ for EUM and $125 \mathrm{hPa}$ for JMA. The maximum pressure ranges between $965 \mathrm{hPa}$ for NWC and $1004 \mathrm{hPa}$ for EUM.

- The percentage of AMVs by layer (with low layer between 700 and $1000 \mathrm{hPa}$, medium layer between 400 and $700 \mathrm{hPa}$, and high layer between 100 and $400 \mathrm{hPa}$ ) are respectively, 23\% to 37\%, $9 \%$ to $20 \%$, and $43 \%$ to $67 \%$. The main outlier of these is NOA at medium levels with only $3 \%$.

Figure 2 shows the spatial localization of AMVs inside the Himawari-8 Full-Disk, and the distribution of parameters (speed, direction, pressure, and CQI), for the different AMV datasets with CQI $>=50 \%$. 


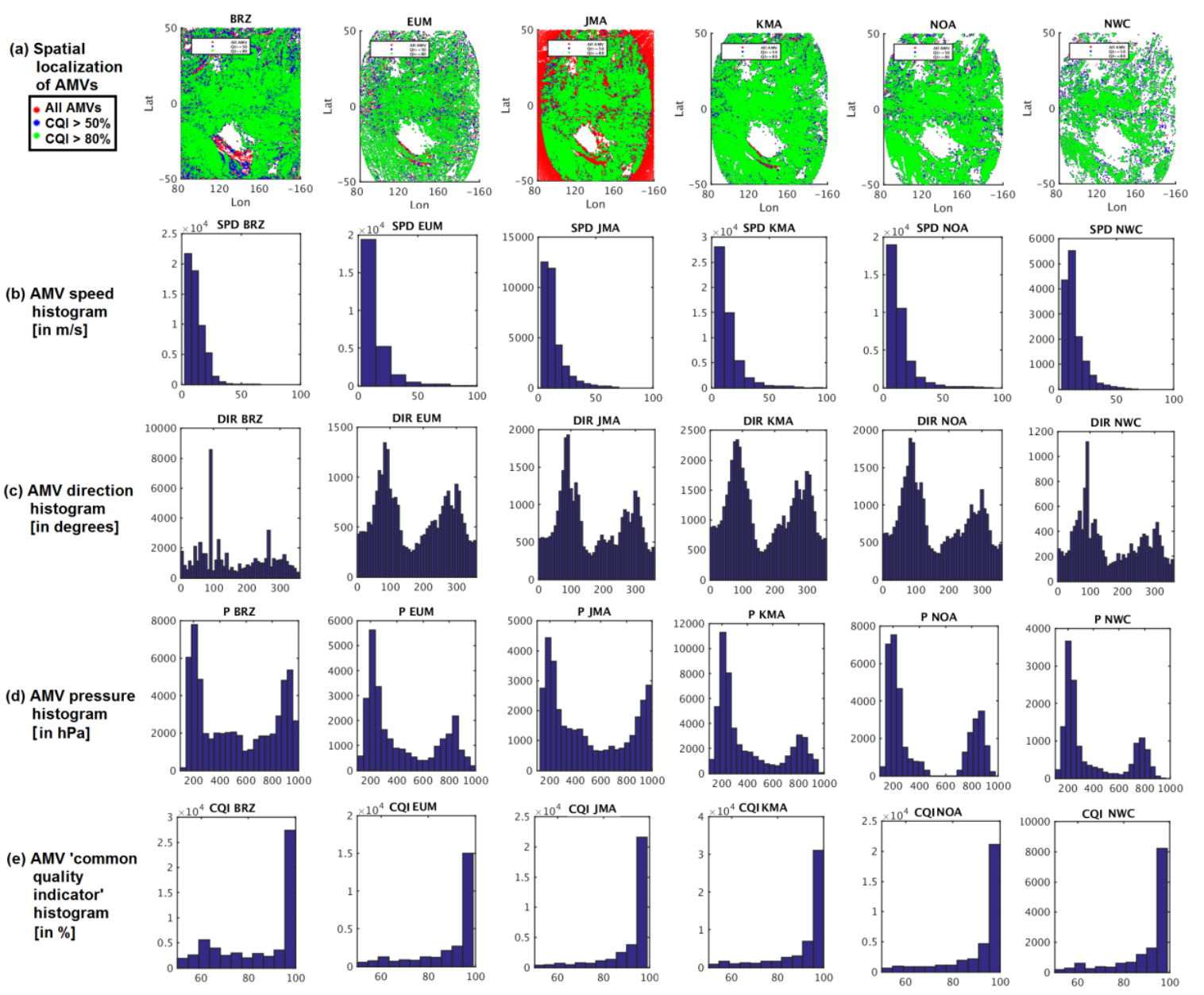

Figure 2. Spatial localization of Atmospheric Motion Vectors (AMVs) (a), and histograms of parameters: (b) AMV speed in $\mathrm{m} \mathrm{s}^{-1}$, (c) AMV direction in degrees, (d) AMV pressure in $\mathrm{hPa}$, (e) AMV CQI (Common Quality Indicator) in \%, for the AMV datasets in Experiment 1 with CQI $>=50 \%$. From left to right, BRZ, EUM, JMA, KMA, NOA, NWC. BRZ: Brazil Center for Weather Prediction and Climate Studies (CPTEC/INPE); EUM: European Organization for the Exploitation of Meteorological Satellites (EUMETSAT); JMA: Japan Meteorological Agency; KMA: Korea Meteorological Administration; NOA: United States National Oceanic and Atmospheric Administration (NOAA); NWC: Satellite Application Facility on Support to Nowcasting (NWCSAF).

The distributions are generally similar for all centers, with much better general agreement than in the previous 'AMV Intercomparison' [15], but with following items to be taken into account:

(1) The distribution of direction values for BRZ shows some directions much more frequent than others, with two anomalous peaks at $90^{\circ}$ and $270^{\circ}$. This issue also partially seems to occur with NWC, with a possible anomalous peak at $90^{\circ}$.

(2) The distribution of the CQI values is rather similar for all centers. This circumstance does not happen when the other quality indicators are considered: the QIF or QINF, for which the calculation results are quite different for the different centers (not shown).

(3) The distribution of AMV pressure values is the most divergent for the different centers, due to the different AMV height assignment methods used; only EUM and NWC being similar because of both using 'CCC method'. 
Figure 3 shows the 'parameter plot' for different variables (speed, direction, pressure, CQI), for the collocated AMVs from the different centers, with CQI $>=50 \%$ and a collocation distance up to $55 \mathrm{~km}$.

(a)

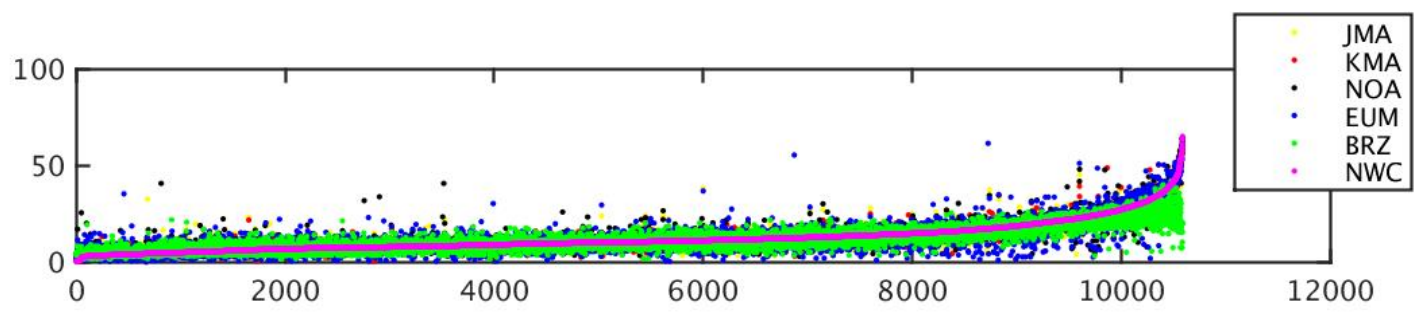

(b)

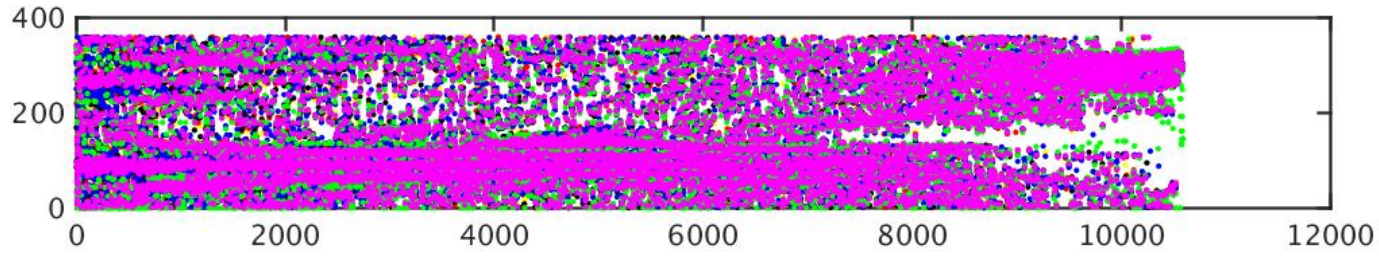

(c)

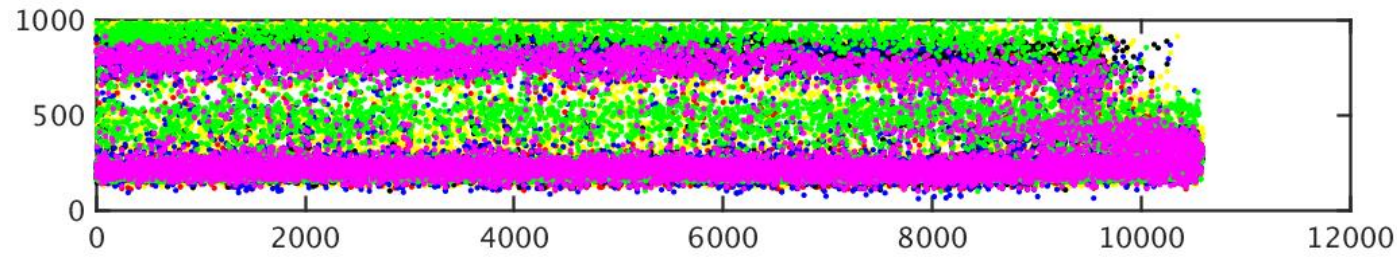

(d)

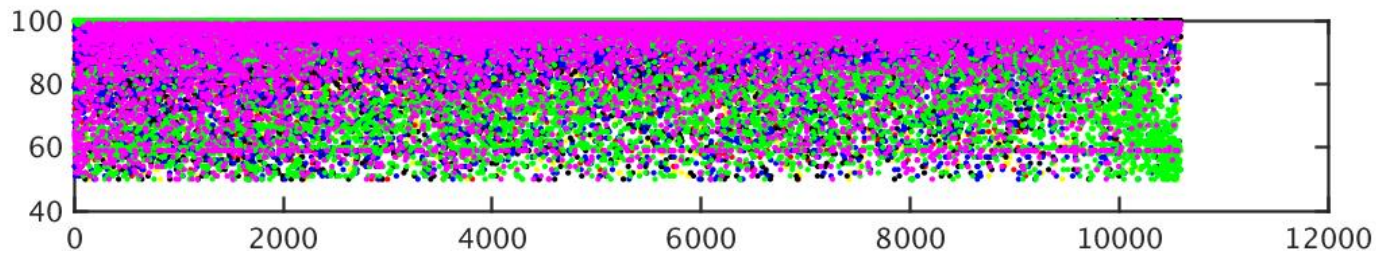

Figure 3. 'Parameter plot' for collocated AMVs from the different AMV datasets in Experiment 1, with CQI $>=50 \%$ and a collocation distance up to $55 \mathrm{~km}$. Considered variables are speed (a), direction (b), pressure (c), and CQI (d). Color codes correspond to BRZ in green, EUM in blue, JMA in yellow, KMA in red, NOA in black, NWC in magenta.

Collocated AMVs in Figure 3 are sorted by increasing speed for NWC. Some specific elements are here significant:

- $\quad$ EUM has many high-speed outliers (blue dots in Figure 3a). BRZ also has a cluster of low-speed outliers (green dots in the far right of the plot). Differences in speed are not significant considering the rest of AMV producers.

- Comparing with the previous 'AMV Intercomparison' [15], the direction (Figure 3b) shows more easterlies even at high speeds, which is likely due to higher speed jets in the southern hemisphere during mid-winter (July), compared to September for that study.

- The pressure (Figure 3c) shows, in general, very few low-level AMVs with speeds greater than $20 \mathrm{~m} \mathrm{~s}^{-1}$. Comparing with the previous 'AMV Intercomparison' [15], there is a large number of high-level AMVs with slow speeds at high levels, for which we cannot offer an explanation.

- $\quad$ Comparing the CQI (Figure 3d) with that for the QINF (not shown), there is more homogeneity between centers. This provides some evidence that the CQI gives better agreement in the AMVs, with a tendency to retain higher CQI values. The generally lower CQI values for BRZ (green dots) might be related to the larger differences in the AMV pressure with the other centers. Some lower CQI values also occur for EUM (blue dots), which might be related to the differences in AMV speeds. 
Figure 4 is a scatterplot of AMV pressures for collocated AMVs from the different centers versus EUM AMV pressures, showing substantial variability depending on the height assignment method used.

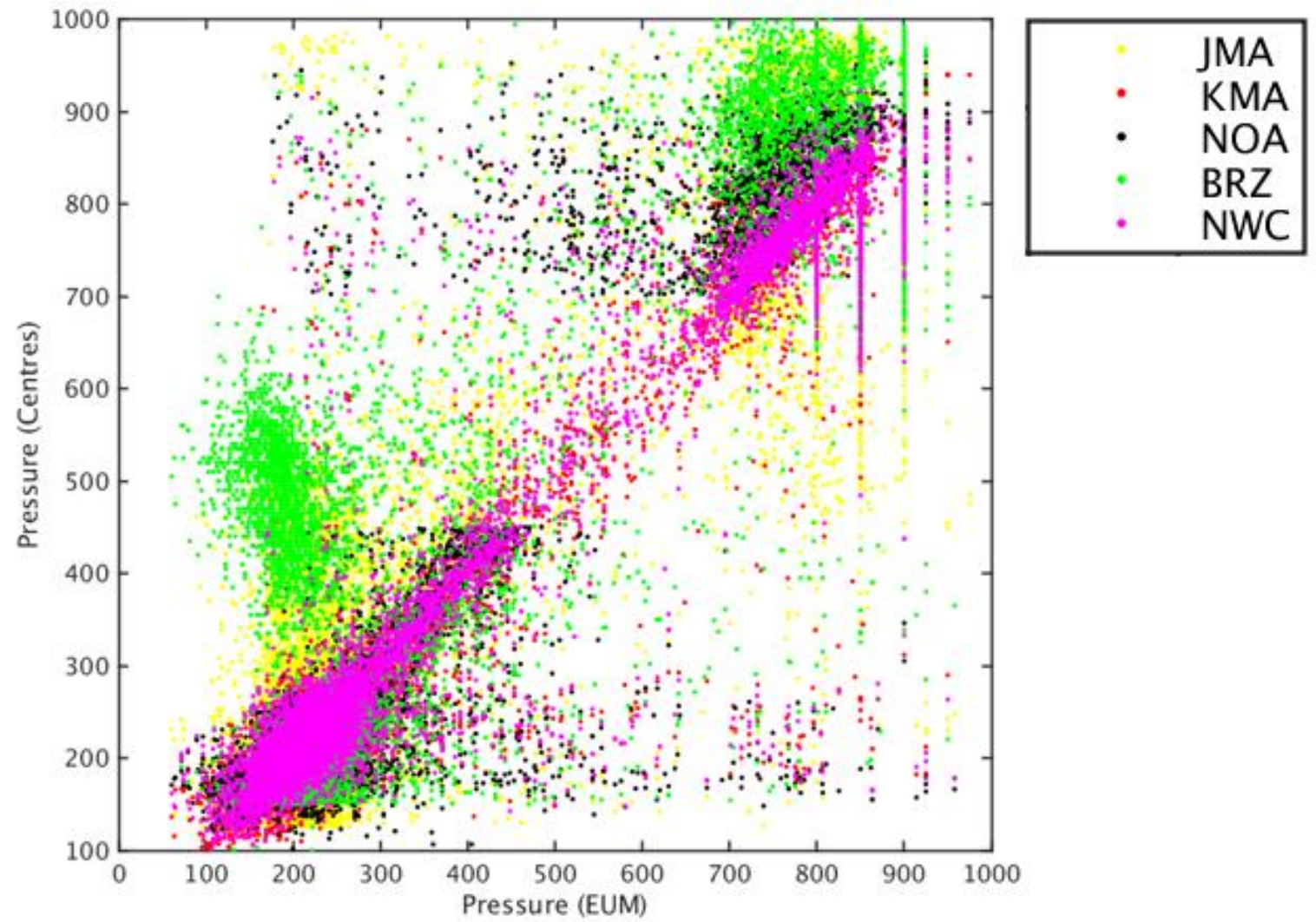

Figure 4. Scatterplot of collocated AMV pressures for Experiment 1, using CQI $>=50 \%$ and a collocation distance up to $55 \mathrm{~km}$, for each center versus EUM AMV pressures (BRZ in green; JMA in yellow; KMA in red; NOA in black; NWC in magenta).

Three of the wind producers (NOA, EUM, NWC) use methods based on the Cloud products, with both EUM and NWC using the 'CCC method' and, therefore, exhibiting the most similarity. This similarity between EUM and NWC is evident in Figure 4, in which the corresponding magenta clusters concentrate in the diagonal. In spite of using the Cloud products, the black dots related to NOA AMVs behave very differently, with no AMVs between 450 and $700 \mathrm{hPa}$ and many AMVs located in a very different layer than the related EUM AMVs.

The remainder of the centers use methods not based on the provided cloud product. Here, the red dots related to KMA AMVs are in relative agreement with EUM AMVs. But BRZ and JMA AMVs show many points plotted away from the diagonal: BRZ AMVs show large clusters of green dots above the diagonal (indicating generally AMV lower in altitude as compared to EUM), and JMA AMVs show many yellow dots located at medium levels (compared to equivalent EUM low-level AMVs) and some located at very low levels (compared to equivalent EUM higher AMVs).

The updated AMV height assignment methods with respect to those in the previous 'AMV Intercomparison' [15], exhibit more variability in the AMV pressure values than the one shown there.

When the AMVs are compared to radiosonde winds (in Table 3 using the CQI threshold of $50 \%$, and in Table 4 using the CQI threshold of $80 \%$ ), the best results are for JMA (with a vector RMS of $6 \mathrm{~m} \mathrm{~s}^{-1}$ ), and then for NWC and NOA (with a vector RMS of 7-8 $\mathrm{m} \mathrm{s}^{-1}$ ). BRZ and EUM show poor results for the low-quality threshold, while much better for the high-quality threshold, for which there is more homogeneity between centers. 
In addition, there are important differences in the number of AMVs for the different centers despite using, all of them, the prescribed configuration.

Table 3. Experiment 1: Comparison of AMVs, with CQI $>=50 \%$, to radiosonde winds within $150 \mathrm{~km}$. $(\mathrm{N}=$ Number of Matches; Pre Bias = Pressure Bias; Pre RMS = Pressure Root Mean Square Error; Spd Bias $=$ Wind Speed Bias; Spd RMS $=$ Wind Speed Root Mean Square Error; Dir Bias = Wind Direction Bias; Vec RMS = Wind Vector Root Mean Square Error). The extremes for each category are highlighted; in yellow, the worst value, and in green, the best value.

\begin{tabular}{cccccccc}
\hline & N & Pre Bias & Pre RMS & Spd Bias & Spd RMS & Dir Bias & Vec RMS \\
\hline BRZ & 774 & 0.90 & 14.68 & -1.28 & 10.00 & -13.13 & 12.61 \\
EUM & 443 & -1.63 & 16.52 & -1.74 & 7.86 & 8.69 & 12.67 \\
JMA & 400 & 0.50 & 13.81 & -0.91 & 3.95 & 1.30 & 5.74 \\
KMA & 859 & -0.55 & 15.16 & -1.88 & 7.61 & 5.46 & 10.10 \\
NOA & 512 & -1.05 & 14.15 & -0.86 & 6.29 & 1.10 & 8.16 \\
NWC & 163 & -0.69 & 15.11 & -1.14 & 4.99 & -1.47 & 6.80 \\
\hline
\end{tabular}

Table 4. The same as Table 3, but with CQI $>=80 \%$.

\begin{tabular}{cccccccc}
\hline & N & Pre Bias & Pre RMS & Spd Bias & Spd RMS & Dir Bias & Vec RMS \\
\hline BRZ & 448 & 0.02 & 13.97 & 0.31 & 6.07 & -14.61 & 8.62 \\
EUM & 312 & -0.83 & 16.54 & -1.79 & 6.54 & 8.31 & 8.56 \\
JMA & 344 & 0.78 & 13.77 & -1.07 & 4.07 & 1.09 & 5.93 \\
KMA & 666 & -0.79 & 15.51 & -1.56 & 6.42 & 2.78 & 8.97 \\
NOA & 427 & -1.49 & 13.96 & -0.89 & 5.42 & 0.45 & 7.52 \\
NWC & 132 & -0.41 & 15.08 & -0.97 & 5.01 & -5.98 & 6.94 \\
\hline
\end{tabular}

Validating the collocated AMVs against the ECMWF ERA-Interim NWP analysis winds, in Table 5 using the QINF threshold of $80 \%$ and in Table 6 using the CQI threshold of $80 \%$, the differences between centers are smaller, and even smaller using the CQI for the filtering. JMA has again the best results, and only BRZ statistics are visibly worse than the rest.

Although not shown, the situation is similar for all AMVs altogether and for the AMVs in the high and the medium layer. At the low layer, BRZ statistics are better, leaving it in an intermediate position and EUM in the last position.

Table 5. Experiment 1: Comparison of collocated AMVs with QINF $>=80 \%$, with NWP analysis winds. ( $\mathrm{N}=$ Number of AMVs; NBF = Number of AMVs with Best-Fit Pressure Value; VD = Vector Difference for all AMVs; VDBF = Vector Difference for AMVs with Best-Fit Pressure Value; RMS = Root Mean Square Error for all AMVs; RMSBF = Root Mean Square Error for AMVs with Best-Fit Pressure Value). The extremes for each category are highlighted; in yellow, the worst value, and in green, the best value.

\begin{tabular}{ccccccc}
\hline & N & NBF & VD & VDBF & RMS & RMSBF \\
\hline BRZ & 4930 & 1191 & 5.90 & 5.27 & 8.47 & 8.16 \\
EUM & 4930 & 1625 & 3.96 & 3.19 & 4.93 & 4.28 \\
JMA & 4930 & 1793 & 2.48 & 2.24 & 2.96 & 2.77 \\
KMA & 4930 & 1732 & 3.69 & 2.85 & 4.61 & 3.79 \\
NOA & 4930 & 1757 & 3.45 & 2.76 & 4.30 & 3.73 \\
NWC & 4930 & 1763 & 3.95 & 3.09 & 4.70 & 3.95 \\
\hline
\end{tabular}


Table 6. Experiment 1: The same as Table 5, but with Common Quality Indicator $>=80 \%$.

\begin{tabular}{ccccccc}
\hline & N & NBF & VD & VDBF & RMS & RMSBF \\
\hline BRZ & 8076 & 2122 & 5.54 & 4.85 & 7.53 & 7.13 \\
EUM & 8076 & 2655 & 4.04 & 3.24 & 4.97 & 4.28 \\
JMA & 8076 & 2860 & 2.59 & 2.33 & 3.10 & 2.89 \\
KMA & 8076 & 2802 & 3.80 & 2.97 & 4.73 & 3.94 \\
NOA & 8076 & 2854 & 3.54 & 2.82 & 4.36 & 3.74 \\
NWC & 8076 & 2791 & 3.99 & 3.17 & 4.74 & 4.03 \\
\hline
\end{tabular}

Table 7 shows an additional comparison of collocated AMVs against the NWP analysis winds, in which a different CQI threshold is defined for each center so that a similar number of AMVs is kept for all of them (at least the 10,000 best AMVs). The CQI threshold ranges from $98 \%$ to $100 \%$ for all the centers except for NWC, for which it is $90 \%$, as the total number of AMVs for NWC is much smaller than for the other centers in this Experiment 1. Despite the different criteria considered for this Table 7, the distribution of errors is not very different to that in Tables 3 and 4: JMA has again the best statistics, with NOA and NWC in later positions. The largest deviations are now being split between BRZ and EUM.

Table 7. Experiment 1: Comparison of all AMVs to NWP analysis winds, with a CQI threshold so that at least 10,000 AMVs are kept. (CQI = Common Quality Indicator Threshold used for each center; $\mathrm{N}=$ Number of AMVs; NBF = Number of AMVs with Best-Fit Pressure Value; VD = Vector Difference for all AMVs; VDBF = Vector Difference for AMVs with Best-Fit Pressure Value; RMS = Root Mean Square Error for all AMVs; RMSBF = Root Mean Square Error for AMVs with Best-Fit Pressure Value). The extremes for each category are highlighted; in yellow, the worst value, and in green, the best value.

\begin{tabular}{cccccccc}
\hline & CQI & N & NBF & VD & VDBF & RMS & RMSBF \\
\hline BRZ & 100 & 18355 & 3946 & 4.71 & 4.14 & 6.62 & 6.21 \\
EUM & 98 & 11120 & 3712 & 4.80 & 3.83 & 6.90 & 6.19 \\
JMA & 99 & 11805 & 4353 & 2.52 & 2.27 & 3.19 & 3.02 \\
KMA & 99 & 19170 & 7166 & 4.74 & 3.63 & 6.57 & 5.65 \\
NOA & 99 & 14086 & 5342 & 3.78 & 2.89 & 5.07 & 4.27 \\
NWC & 90 & 10117 & 3669 & 4.25 & 3.28 & 5.21 & 4.31 \\
\hline
\end{tabular}

The difference of the AMV pressure level with the AMV best-fit pressure level is considered in Figure 5. The best-fit pressure level is computed for each AMV according to the method described by Salonen et al. (2012) [19].

This method defines the pressure for which the background NWP model wind has a minimum vector difference with the AMV wind. It does this by first determining the NWP model pressure level with a minimum vector difference between the AMV wind and the NWP wind.

Then, the actual minimum is calculated by using a parabolic fit with the vector difference for this model level and the levels directly above and below, which must be both less than or equal to $4 \mathrm{~m}$ $\mathrm{s}^{-1}$, and at least $2 \mathrm{~m} \mathrm{~s}^{-1}$ smaller than the vector differences more than $100 \mathrm{hPa}$ away from the best-fit pressure level. Therefore, this method is dependent on the model vertical resolution.

Similar to previous studies, the number of best-fit pressure level matches is for about $30 \%$ of the AMVs: depending on the AMV dataset, $21 \%$ to $36 \%$ of the AMVs are adjusted to a best-fit pressure.

Results show an approximate Gaussian distribution of the variable 'AMV best-fit pressure level-AMV pressure level' for all centers, which is more consistent than the one found in the previous 'AMV Intercomparison' [15]. 


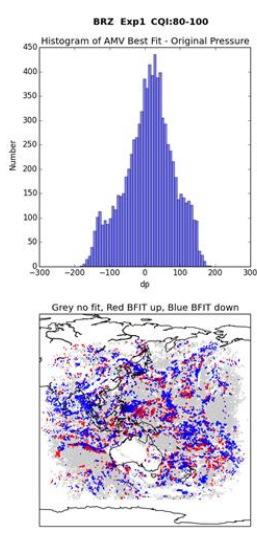

(a) BRZ

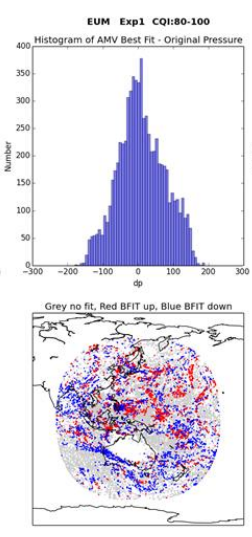

(b) EUM

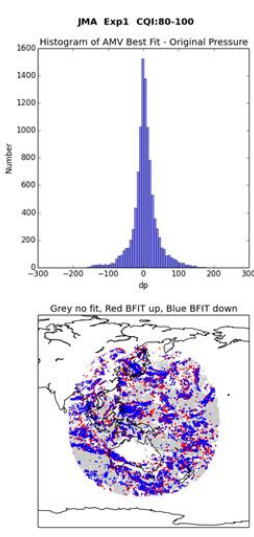

(c) JMA

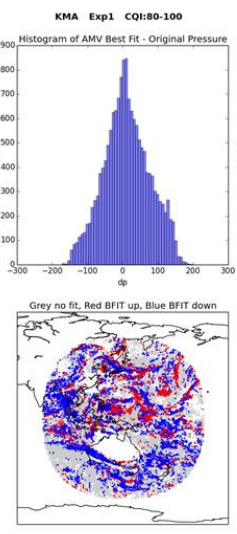

(d) KMA

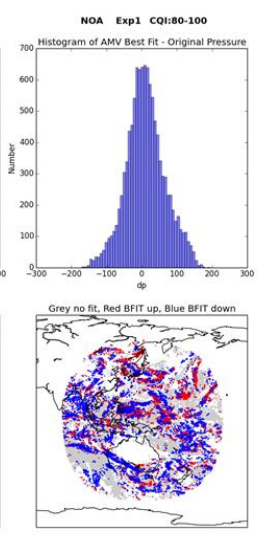

(e) NOA

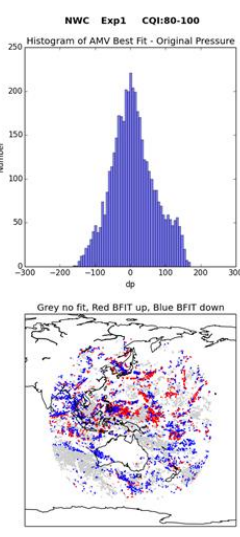

(f) NWC

Figure 5. Histogram (upper) and maps (lower) of the difference 'AMV best-fit pressure level-AMV pressure level' for (a) BRZ, (b) EUM, (c) JMA, (d) KMA, (e) NOA, (f) NWC. In the maps, red shows the best-fit pressure at a higher level; blue shows the best-fit pressure at a lower level.

The pressure difference is centered near zero (upper panels in Figure 5) and extends up to $\pm 200 \mathrm{hPa}$. The exception to this is JMA, for which the deviation is up to $\pm 100 \mathrm{hPa}$ only. This way, it is clear that JMA AMVs are much nearer to the AMV best-fit pressure level than all other datasets. Although this might also indicate that the JMA AMV height assignment method has a stronger dependency on the NWP model background.

The maps in the lower panels of Figure 5 depict the best-fit displacements above (red) and below (blue) the AMV level for each AMV, which tend to be in similar locations for all centers for collocated AMVs.

In the northern hemisphere, generally, only high-level AMVs are adjusted, while, in the southern hemisphere, both high- and low-level clouds are adjusted to the best-fit level.

An additional calculation is defined to evaluate the difference between the AMV wind (before and after the best-fit pressure level correction) and the NWP model wind. Results are shown in Tables 8 and 9 for all AMVs for which the best-fit pressure level correction could be applied, using, respectively, a QINF threshold of $80 \%$ and a CQI threshold of $80 \%$.

The speed bias is reduced in the case of BRZ, due to the large value of this parameter before the best-fit pressure level correction. For the rest of the centers, the speed bias is already small before the correction, and so the impact of this correction on this parameter is not significant.

Finally, the speed standard deviation is reduced about $70 \%-75 \%$ after the correction for all centers except for JMA, for which the reduction is approximately $38 \%$, as the JMA standard deviation before the best-fit level correction is nearly as good as for the other centers after the best-fit level correction.

Table 8. Experiment 1: Speed Bias (BIAS) and Speed Standard Deviation (STD) with respect to the NWP analysis winds, before and after the best-fit pressure level correction, for all AMVs for which the best-fit pressure level correction could be applied, with QINF $>=80 \%$. The extremes for each category are highlighted; in yellow, the worst value, and in green, the best value.

\begin{tabular}{ccccc}
\hline & BIAS before & STD before & BIAS after & STD after \\
\hline BRZ & -1.00 & 4.38 & 0.28 & 1.16 \\
EUM & -0.15 & 4.65 & 0.26 & 1.19 \\
JMA & 0.06 & 1.79 & 0.21 & 1.11 \\
KMA & -0.12 & 4.42 & 0.21 & 1.14 \\
NOA & -0.04 & 3.77 & 0.23 & 1.17 \\
NWC & 0.13 & 3.97 & 0.27 & 1.18 \\
\hline
\end{tabular}


Table 9. The same as Table 8 , but with CQI $>=80 \%$.

\begin{tabular}{ccccc}
\hline & BIAS before & STD before & BIAS after & STD after \\
\hline BRZ & -1.12 & 4.33 & 0.25 & 1.16 \\
EUM & -0.20 & 4.57 & 0.26 & 1.18 \\
JMA & 0.05 & 1.78 & 0.22 & 1.11 \\
KMA & -0.33 & 4.55 & 0.21 & 1.14 \\
NOA & -0.04 & 3.77 & 0.23 & 1.17 \\
NWC & -0.25 & 4.01 & 0.23 & 1.16 \\
\hline
\end{tabular}

\subsection{Experiment 2}

In this case, AMV producers extracted cloudy AMVs with the Himawari-8/AHI IR10.4 $\mu$ m triplet 1200-1220 UTC, using their best options for the AMV calculation, and their specific configuration for target box sizes, search scene sizes, and target locations. This way, differences of each AMV extraction process with respect to the previous prescribed configuration can be compared.

Table 10 shows the parameter distribution for the AMV datasets, with a CQI threshold of $50 \%$. As in Experiment 1, this threshold is used in all AMV datasets to avoid the processing of 'low-quality AMVs'; in some elements of the Intercomparison, an additional CQI threshold of $80 \%$ is used to verify its impact on the improvement of the validation parameters in the different AMV datasets.

Table 10. Parameter distribution for the different AMV datasets in experiment 2 with CQI >=50\%.

\begin{tabular}{|c|c|c|c|c|c|c|}
\hline & BRZ & EUM & JMA & KMA & NOA & NWC \\
\hline Total Number of AMVs & 74,100 & 30,806 & 67,324 & 53,171 & 47,320 & 146,780 \\
\hline Common Quality Index $>=50 \%$ & 65,673 & 28,174 & 27,057 & 47,411 & 45,782 & 146,496 \\
\hline Speed $\min .(\mathrm{m} / \mathrm{s})$ & 2.55 & 2.50 & 2.51 & 2.50 & 3.00 & 2.50 \\
\hline Speed max. (m/s) & 70.06 & 145.55 & 85.94 & 97.01 & 94.16 & 84.03 \\
\hline Speed mean $(\mathrm{m} / \mathrm{s})$ & 12.02 & 13.37 & 14.00 & 13.89 & 13.97 & 12.97 \\
\hline Pressure min. (hPa) & 100.00 & 57.58 & 125.02 & 106.81 & 105.72 & 101.00 \\
\hline Pressure max. (hPa) & 1000.00 & 1009.80 & 996.47 & 1000.00 & 987.20 & 972.50 \\
\hline Pressure mean $(\mathrm{hPa})$ & 487.06 & 438.03 & 497.74 & 450.50 & 432.01 & 409.12 \\
\hline Percentage of Low AMVs (\%) & 29.96 & 27.15 & 32.27 & 24.56 & 33.55 & 25.63 \\
\hline Percentage of Mid AMVs (\%) & 21.19 & 14.14 & 17.51 & 22.02 & 2.56 & 11.52 \\
\hline Percentage of High AMVs (\%) & 48.85 & 58.71 & 50.22 & 53.42 & 63.89 & 62.86 \\
\hline Speed min. of Low AMVs (m/s) & 2.55 & 2.50 & 2.51 & 2.50 & 3.01 & 2.50 \\
\hline Speed max. of Low AMVs (m/s) & 46.46 & 54.21 & 32.67 & 70.27 & 37.59 & 36.22 \\
\hline Speed mean of Low AMVs (m/s) & 9.39 & 8.89 & 9.14 & 10.54 & 9.77 & 8.76 \\
\hline Pressure min. of Low AMVs (hPa) & 700.00 & 700.06 & 700.04 & 700.00 & 700.08 & 700.00 \\
\hline Pressure max. of Low AMVs (hPa) & 1000.00 & 1009.80 & 996.47 & 1000.00 & 987.20 & 972.50 \\
\hline Pressure mean of Low AMVs (hPa) & 870.56 & 820.69 & 887.37 & 817.99 & 835.02 & 788.38 \\
\hline Speed min. of Mid AMVs (m/s) & 2.55 & 2.51 & 2.51 & 2.50 & 3.04 & 2.51 \\
\hline Speed max. of Mid AMVs (m/s) & 69.46 & 69.67 & 72.05 & 80.01 & 69.25 & 62.00 \\
\hline Speed mean of Mid AMVs (m/s) & 13.73 & 13.95 & 14.46 & 15.11 & 21.30 & 14.95 \\
\hline Pressure min. of Mid AMVs (hPa) & 400.03 & 400.06 & 400.00 & 400.02 & 400.06 & 400.50 \\
\hline Pressure max. of Mid AMVs (hPa) & 699.97 & 699.73 & 699.97 & 699.98 & 449.98 & 699.50 \\
\hline Pressure mean of Mid AMVs (hPa) & 528.14 & 529.96 & 533.05 & 514.73 & 426.70 & 535.47 \\
\hline Speed min. of High AMVs (m/s) & 2.59 & 2.50 & 2.52 & 2.50 & 3.00 & 2.50 \\
\hline Speed max. of High AMVs (m/s) & 70.06 & 145.55 & 85.94 & 97.01 & 94.16 & 84.03 \\
\hline Speed mean of High AMVs (m/s) & 12.88 & 15.31 & 16.97 & 14.92 & 15.88 & 14.33 \\
\hline Pressure min. of High AMVs (hPa) & 100.00 & 57.58 & 125.02 & 106.81 & 105.72 & 101.00 \\
\hline Pressure max. of High AMVs (hPa) & 400.00 & 399.94 & 399.98 & 400.00 & 399.97 & 400.00 \\
\hline Pressure mean of High AMVs (hPa) & 233.99 & 238.96 & 235.09 & 255.08 & 220.57 & 231.35 \\
\hline
\end{tabular}

The differences with Experiment 1 are caused by the specific configuration of each center for the target box sizes, search scene sizes, and target locations: 
- The total number of AMVs ranges from 31,000 to 147,000, with the lower values for EUM and the larger values for NWC, and a factor of five between the numbers of AMVs of both centers.

- Comparing with Experiment 1, the difference in the total number of AMVs is small for all centers (only up to 25\%) except for NWC, for which the total number of AMVs is one order of magnitude larger. The main reason for this big increment in the number of NWC AMVs is its high density of tracers in its operational specific conditions (with the smallest nominal separation between tracers of all methods).

- The number of AMVs is much larger with Himawari satellites than in the previous 'AMV Intercomparison' with MSG satellite [15], which found up to approximately 10,000 AMVs (although KMA was then able to calculate 50,000 AMVs and NWC was able to calculate 90,000 AMVs). As already said, this is due, in part, to the higher resolution of Himawari/AHI $(2 \mathrm{~km})$ versus MSG/SEVIRI (3 km), more oceanic regions, and fewer deserts.

- The maximum speed ranges from $70 \mathrm{~m} \mathrm{~s}^{-1}$ (BRZ) to $97 \mathrm{~m} \mathrm{~s}^{-1}$ (KMA), with outliers of $146 \mathrm{~m} \mathrm{~s}^{-1}$ again for EUM.

- The minimum pressure ranges between $57 \mathrm{hPa}$ for EUM and $125 \mathrm{hPa}$ for JMA. The maximum pressure ranges between $972 \mathrm{hPa}$ for NWC and $1010 \mathrm{hPa}$ for EUM.

- The percentage of AMVs by layer (with the low layer between 700 and $1000 \mathrm{hPa}$, the medium layer between 400 and $700 \mathrm{hPa}$, and the high layer between 100 and $400 \mathrm{hPa}$ ) are, respectively, $25 \%$ to $34 \%, 12 \%$ to $22 \%$, and $49 \%$ to $64 \%$. The main outlier of these is NOA again at medium levels with only $3 \%$.

Figure 6 shows the spatial localization of AMVs inside the Himawari-8 Full-Disk, and the distribution of parameters (speed, direction, pressure, and CQI), for the different AMV datasets with CQI $>=50 \%$.

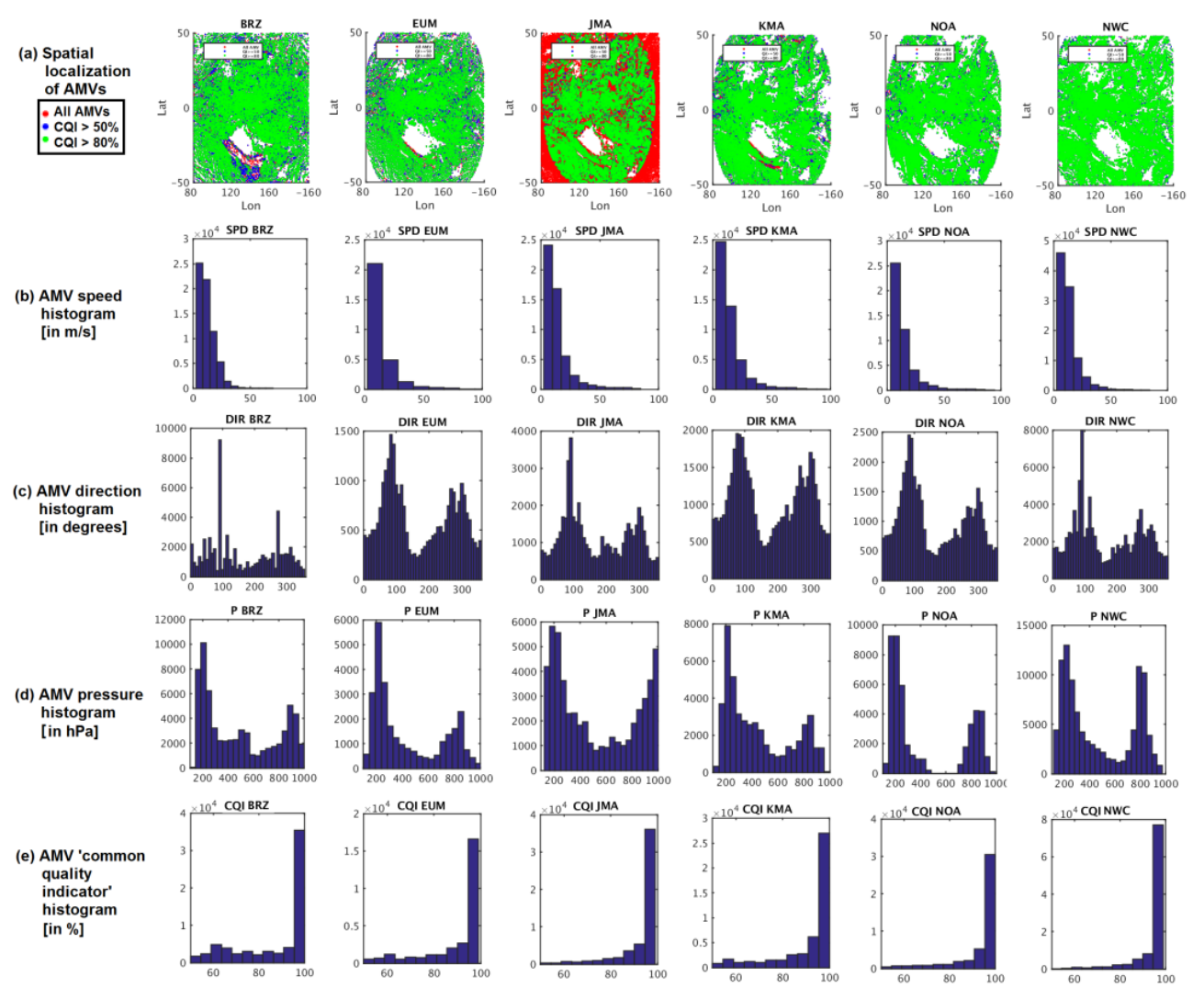

Figure 6. Spatial localization of AMVs (a), and histograms of parameters: (b) AMV speed in $\mathrm{m} \mathrm{s}^{-1}$, (c) AMV direction in degrees, (d) AMV pressure in $\mathrm{hPa}$, (e) AMV CQI in \%, for the AMV datasets in Experiment 2 with CQI $>=50 \%$. From left to right, BRZ, EUM, JMA, KMA, NOA, NWC. 
The parameter distributions are very similar to those for Experiment 1 in Figure 2, and so the same conclusions apply. Specifically, the distribution of the CQI values appear similar for all centers again. The differences in the height assignment process drive again the majority of differences observed in the AMV datasets.

Figure 7 shows the 'parameter plot' for different variables (speed, direction, pressure, CQI), for the collocated AMVs from the different centers, with CQI $>=50 \%$ and a collocation distance up to $55 \mathrm{~km}$.

(a)

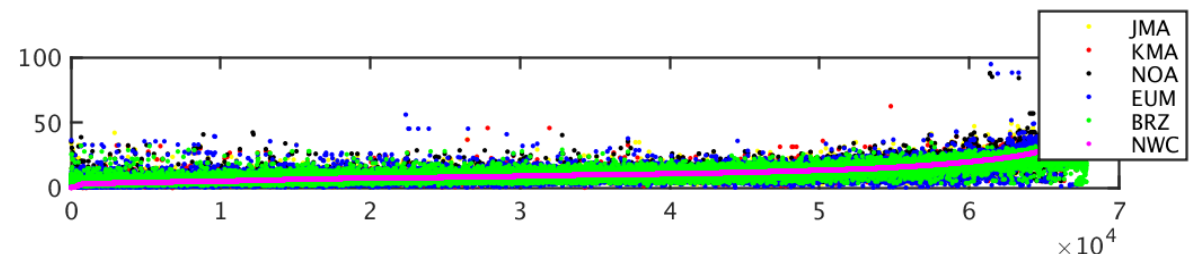

(b)

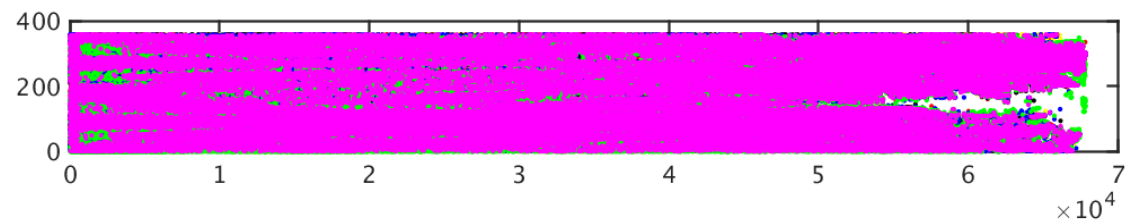

(c)

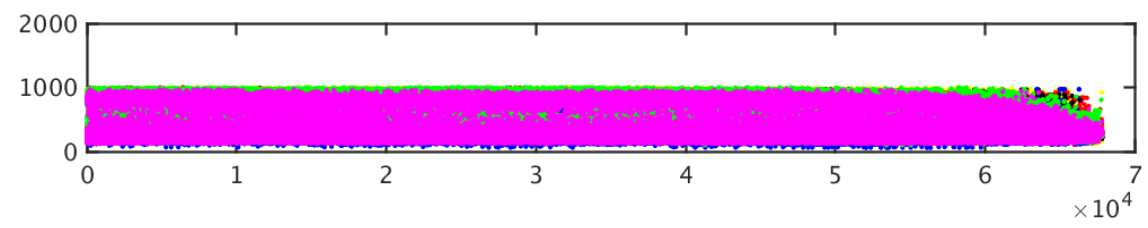

(d)

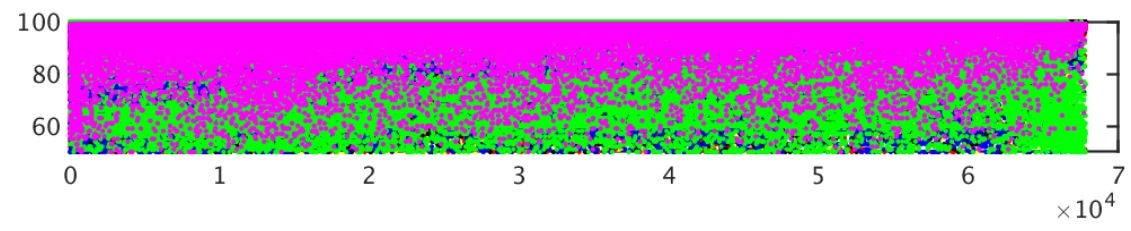

Figure 7. 'Parameter plot' for collocated AMVs from the different AMV datasets in Experiment 2, with CQI $>=50 \%$ and a collocation distance up to $55 \mathrm{~km}$. Considered variables are speed (a), direction (b), pressure (c), and CQI (d). Color codes correspond to BRZ in green, EUM in blue, JMA in yellow, KMA in red, NOA in black, NWC in magenta.

Collocated AMVs in Figure 7 are sorted by increasing speed for NWC. In general, Figure 7 is in agreement with Figure 3 for Experiment 1 of this study:

- $\quad$ The speed plot in Figure 7a shows that the EUM high-speed outliers (blue dots) and the BRZ cluster of low-speed outliers (green dots in the far right of the plot), detected in Experiment 1, are seen again.

- The CQI plot in Figure 7d shows that there is again a tendency to retain high CQI values for all centers except BRZ, and in some cases also EUM. Their lower CQI values (green and blue dots in the plot) might again be related to the differences in AMV pressure and AMV speeds, respectively, with the other centers.

The other graphs in Figure 7 are, however, dominated by the nearly 150,000 AMVs from NWC, because of which additional information cannot be extracted.

Figure 8 shows the scatterplot of AMV pressures for collocated AMVs from the different centers versus EUM AMV pressures. 


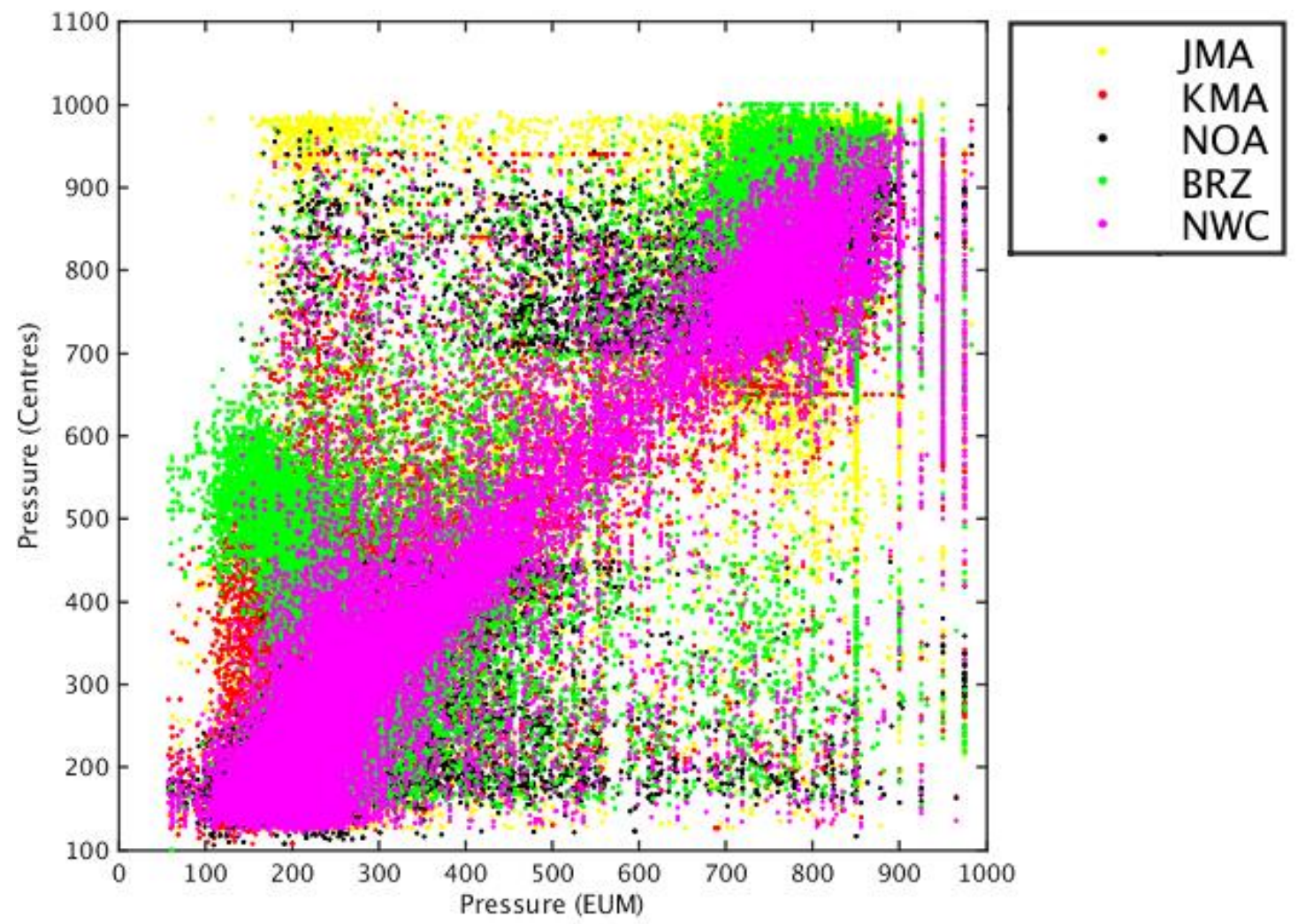

Figure 8. Scatterplot of collocated AMV pressures for Experiment 2, with CQI $>=50 \%$ and a collocation distance up to $55 \mathrm{~km}$, for each center versus EUM AMV pressures (BRZ in green; JMA in yellow; KMA in red; NOA in black; NWC in magenta).

Figure 8 is in agreement with Figure 4 for Experiment 1 of this study, but with a much larger number of AMV collocations between centers.

There is again an excellent agreement between EUM and NWC (magenta clusters), and many points plotted away from the diagonal (from the other centers). NOA has again no AMVs between 450 and $700 \mathrm{hPa}$, and many of its AMVs are away from the diagonal (at both higher and lower altitudes).

There are again two clusters of green dots related to BRZ above the diagonal (with AMVs lower in altitude as compared to EUM), although now there are also some green dots related to BRZ below the diagonal (at higher altitudes). KMA AMVs also show now a cluster of red dots above the diagonal at high levels (lower in altitude).

Finally, JMA AMVs show the same behavior than in Experiment 1, with many yellow dots located at medium levels (compared to equivalent EUM low-level AMVs) and some located at very low levels (compared to equivalent EUM higher AMVs).

When the AMVs are compared to radiosonde winds (in Table 11 using the threshold of $50 \%$, and in Table 12 using the threshold of $80 \%$ for the CQI), the best results are again for JMA (with a vector RMS of $6 \mathrm{~m} \mathrm{~s}^{-1}$ ), and then for NOA and NWC (with a vector RMS of 7-8 $\mathrm{m} \mathrm{s}^{-1}$ ). 
Table 11. Experiment 2: Comparison of AMVs, with CQI $>=50 \%$, to radiosonde winds within 150 $\mathrm{km}$. (N = Number of Matches; Pre Bias = Pressure Bias; Pre RMS = Pressure Root Mean Square Error; Spd Bias = Wind Speed Bias; Spd RMS = Wind Speed Root Mean Square Error; Dir Bias = Wind Direction Bias; Vec RMS = Wind Vector Root Mean Square Error). The extremes for each category are highlighted; in yellow, the worst value, and in green, the best value.

\begin{tabular}{cccccccc}
\hline & N & Pre Bias & Pre RMS & Spd Bias & Spd RMS & Dir Bias & Vec RMS \\
\hline BRZ & 942 & 1.46 & 14.44 & -2.69 & 11.65 & -9.48 & 15.22 \\
EUM & 508 & -0.62 & 15.92 & -2.17 & 7.03 & 10.08 & 8.87 \\
JMA & 313 & -2.94 & 18.33 & -1.36 & 4.64 & -0.83 & 6.34 \\
KMA & 797 & -0.64 & 14.80 & -1.55 & 7.78 & -1.41 & 10.03 \\
NOA & 691 & -1.58 & 13.93 & -0.90 & 5.44 & 1.89 & 7.62 \\
NWC & 2204 & -1.02 & 16.30 & -2.17 & 6.03 & 0.40 & 7.85 \\
\hline
\end{tabular}

Table 12. The same as Table 11, but with Common Quality indicator (CQI) $>=80 \%$.

\begin{tabular}{cccccccc}
\hline & N & Pre Bias & Pre RMS & Spd Bias & Spd RMS & Dir Bias & Vec RMS \\
\hline BRZ & 619 & 1.16 & 13.44 & -0.40 & 7.36 & -14.65 & 9.80 \\
EUM & 366 & -0.66 & 14.74 & -2.20 & 6.15 & 8.43 & 8.56 \\
JMA & 270 & -3.43 & 18.67 & -1.40 & 4.64 & -0.83 & 5.93 \\
KMA & 628 & -0.84 & 14.30 & -1.21 & 7.39 & -2.66 & 8.97 \\
NOA & 599 & -1.69 & 13.98 & -0.88 & 5.25 & 0.39 & 7.52 \\
NWC & 2063 & -1.19 & 16.19 & -2.11 & 5.99 & 0.79 & 6.94 \\
\hline
\end{tabular}

EUM results are much better in Experiment 2, using their specific configuration. The higher quality threshold in Table 12 contributes again to more homogeneity in the statistics of the different centers.

Validating the collocated AMVs against the ECMWF ERA-Interim NWP analysis winds, in Table 13 with QINF >= 80\%, and in Table 14 with CQI >= 80\%, differences between centers are smaller.

Table 13. Experiment 2: Comparison of collocated AMVs, with QINF $>=80 \%$, to NWP analysis winds. $(\mathrm{N}=$ Number of AMVs; NBF = Number of AMVs with Best-Fit Pressure Value; VD = Vector Difference for all AMVs; VDBF = Vector Difference for AMVs with Best-Fit Pressure Value; RMS = Root Mean Square Error for all AMVs; RMSBF = Root Mean Square Error for AMVs with Best-Fit Pressure Value. The extremes for each category are highlighted; in yellow, the worst value, and in green, the best value.

\begin{tabular}{ccccccc}
\hline & N & NBF & VD & VDBF & RMS & RMSBF \\
\hline BRZ & 43281 & 9814 & 5.60 & 5.01 & 8.20 & 7.90 \\
EUM & 43281 & 13270 & 3.84 & 3.05 & 4.96 & 4.24 \\
JMA & 43281 & 14572 & 2.20 & 1.99 & 2.71 & 2.52 \\
KMA & 43281 & 12709 & 3.75 & 3.08 & 5.05 & 4.51 \\
NOA & 43281 & 13765 & 3.41 & 2.74 & 4.26 & 3.64 \\
NWC & 43281 & 13588 & 3.45 & 2.79 & 4.13 & 3.54 \\
\hline
\end{tabular}

Table 14. Experiment 2: The same as Table 13, but with CQI $>=80 \%$.

\begin{tabular}{ccccccc}
\hline & N & NBF & VD & VDBF & RMS & RMSBF \\
\hline BRZ & 56515 & 13075 & 5.73 & 5.11 & 8.35 & 8.02 \\
EUM & 56515 & 17533 & 4.00 & 3.17 & 5.17 & 4.43 \\
JMA & 56515 & 19208 & 2.27 & 2.06 & 2.80 & 2.62 \\
KMA & 56515 & 16635 & 3.92 & 3.23 & 5.25 & 4.72 \\
NOA & 56515 & 18163 & 3.53 & 2.84 & 4.42 & 3.80 \\
NWC & 56515 & 17860 & 3.55 & 2.87 & 4.24 & 3.65 \\
\hline
\end{tabular}


In Tables 13 and 14, JMA has again the best results, and only BRZ statistics are visibly worse. Although not shown, the situation is similar for all AMVs altogether and for the AMVs in the high and the medium layer. At the low layer, BRZ statistics are better, leaving it in an intermediate position and leaving KMA in the last position.

Table 15 shows an additional comparison of collocated AMVs against the NWP analysis winds, in which a different CQI threshold is defined for each center so that a similar number of AMVs is kept for all of them (at least the 10,000 best AMVs). The CQI threshold ranges from $98 \%$ to $100 \%$ for all the centers. Despite the different criteria considered for Table 15, the distribution of errors is similar to those in Tables 11-14: JMA has again the best statistics, with NOA and NWC in second positions. The largest deviations are related again to BRZ.

Table 15. Experiment 2: Comparison of all AMVs to NWP analysis winds, with a CQI threshold so that at least 10,000 AMVs are kept. (CQI = Common Quality Indicator Threshold used for each center; $\mathrm{N}=$ Number of AMVs; NBF = Number of AMVs with Best-Fit Pressure Value; VD = Vector Difference for all AMVs; VDBF = Vector Difference for AMVs with Best-Fit Pressure Value; RMS = Root Mean Square Error for all AMVs; RMSBF = Root Mean Square Error for AMVs with Best-Fit Pressure Value). The extremes for each category are highlighted; in yellow, the worst value, and in green, the best value.

\begin{tabular}{cccccccc}
\hline & CQI & N & NBF & VD & VDBF & RMS & RMSBF \\
\hline BRZ & 100 & 26341 & 5733 & 5.90 & 5.22 & 8.32 & 7.91 \\
EUM & 98 & 12407 & 4056 & 4.43 & 3.46 & 6.13 & 5.28 \\
JMA & 98 & 10595 & 3991 & 2.52 & 2.29 & 3.20 & 3.03 \\
KMA & 99 & 16721 & 5934 & 4.66 & 3.75 & 6.71 & 6.06 \\
NOA & 100 & 14484 & 5484 & 3.84 & 2.91 & 5.06 & 4.21 \\
NWC & 99 & 80625 & 28532 & 4.24 & 3.26 & 5.24 & 4.36 \\
\hline
\end{tabular}

The difference of the AMV pressure level with the AMV best-fit pressure level, computed as described in Figure 5, is considered now in Figure 9. Similarly, the number of best-fit pressure level matches is for $22 \%$ to $38 \%$ of the AMVs. Results show again the approximate Gaussian distribution of the variable 'AMV best-fit pressure level-AMV pressure level' for all centers.

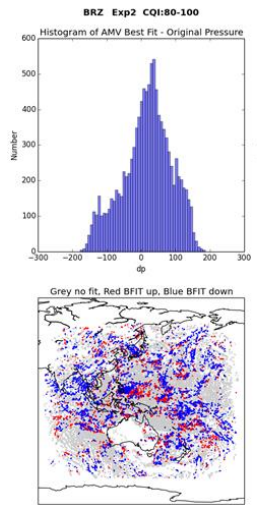

(a) BRZ

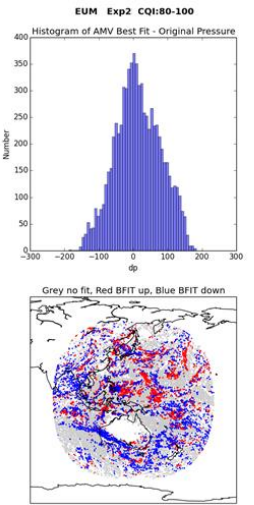

(b) EUM

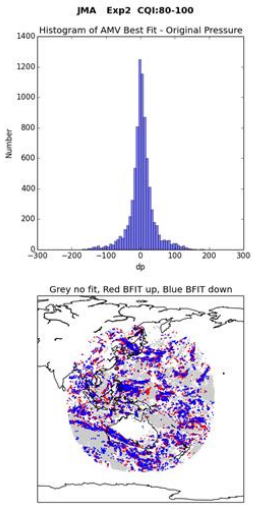

(c) JMA

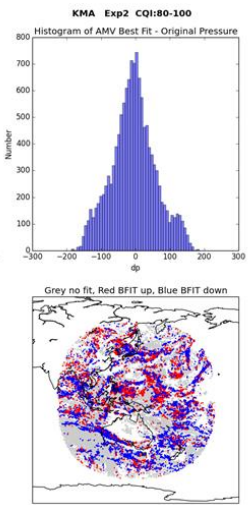

(d) KMA

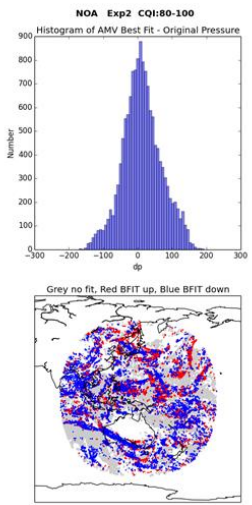

(e) NOA

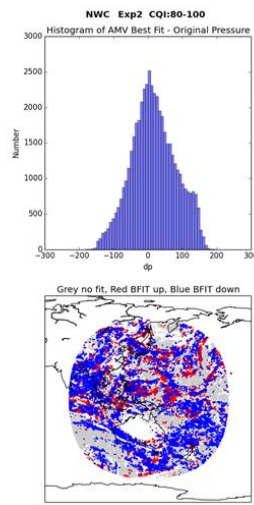

(f) NWC

Figure 9. Histogram (upper) and maps (lower) of the difference 'AMV best-fit pressure level-AMV pressure level' for (a) BRZ, (b) EUM, (c) JMA, (d) KMA, (e) NOA, (f) NWC. In the maps, red shows the best-fit pressure at a higher level; blue shows the best-fit pressure at a lower level.

The pressure difference is centered again near zero (upper panels in Figure 9), extending up to $\pm 200 \mathrm{hPa}$, except for JMA for which the deviation is up to $\pm 100 \mathrm{hPa}$ only. This way, it is again clearer that JMA AMVs are much closer to the AMV best-fit level than all other datasets, although with a possibly stronger dependency on the NWP model background. The maps in the lower panels of 
Figure 9 depict anew the best-fit displacements above (red) and below (blue) the AMV level for each AMV. Again, they tend to be in similar locations for all centers for collocated AMVs.

An additional calculation to evaluate the difference between the AMV wind (before and after the best-fit pressure level correction) and the NWP model wind is defined again. Results are shown in Tables 16 and 17 for all AMVs, for which the 'best-fit pressure level correction' could be applied, using, respectively, the threshold of $80 \%$ for the QINF and the CQI.

Table 16. Experiment 2: Speed Bias (BIAS) and Speed Standard Deviation (STD) with respect to the NWP analysis winds, before and after the best-fit pressure level correction, for all AMVs for which the best-fit pressure level correction could be applied with QINF $>=80 \%$. The extremes for each category are highlighted; in yellow, the worst value, and in green, the best value.

\begin{tabular}{ccccc}
\hline & BIAS before & STD before & BIAS after & STD after \\
\hline BRZ & -1.51 & 4.57 & 0.26 & 1.17 \\
EUM & -0.28 & 4.60 & 0.18 & 1.17 \\
JMA & 0.09 & 1.82 & 0.20 & 1.14 \\
KMA & 0.65 & 3.89 & 0.23 & 1.14 \\
NOA & -0.22 & 3.82 & 0.24 & 1.15 \\
NWC & -0.32 & 3.94 & 0.20 & 1.14 \\
\hline
\end{tabular}

Table 17. The same as Table 16, but with CQI $>=80 \%$.

\begin{tabular}{ccccc}
\hline & BIAS before & STD before & BIAS after & STD after \\
\hline BRZ & -1.57 & 4.56 & 0.26 & 1.16 \\
EUM & -0.20 & 4.57 & 0.26 & 1.18 \\
JMA & 0.07 & 1.82 & 0.21 & 1.12 \\
KMA & 0.60 & 3.97 & 0.22 & 1.15 \\
NOA & -0.22 & 3.82 & 0.24 & 1.15 \\
NWC & -0.68 & 4.10 & 0.18 & 1.12 \\
\hline
\end{tabular}

The speed bias is reduced in the cases of BRZ, KMA, and NWC. For the rest of the centers, the speed bias is already small before the correction, and so the impact of this correction on this parameter is not significant.

Considering the speed standard deviation, again it is reduced around $70 \%-75 \%$ after the correction for all centers except for JMA, for which the reduction is around 38\%, as the JMA standard deviation before the best-fit level correction is nearly as good as for the other centers after the best-fit level correction.

\subsection{Experiment 3}

In this case, producers extracted AMVs with the Himawari-8/AHI infrared $10.4 \mu \mathrm{m}$ triplet for 21 July 2016 0530-0550 UTC, using their best options for the AMV calculation and their specific configurations for target box size, search scene size, and target location (as in Experiment 2).

This dataset is used for validation against NASA's CALIPSO (Cloud-Aerosol Lidar and Infrared Pathfinder Satellite Observation), which provides an independent measurement of cloud top height.

CALIPSO is a line-of-site measurement, so there are few collocations with AMVs (tens of matches only). Therefore, this evaluation is qualitative, as illustrated in the following Figures 10 and 11. The thresholds for determining collocations are a distance of 0.1 degrees for latitude and longitude and a time of one hour. 


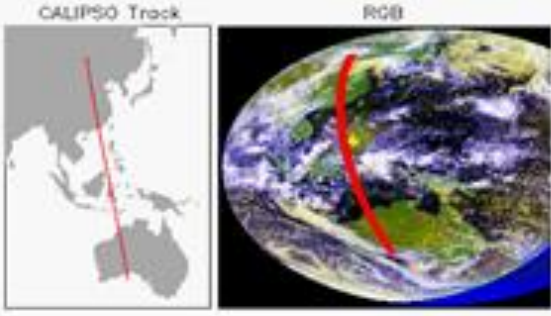

(a) (b)

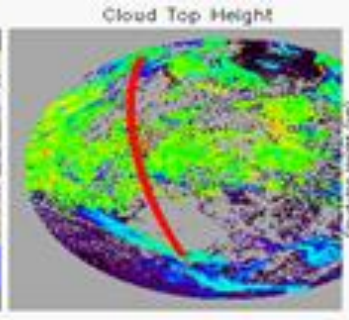

(c)
Stote: Count, Ascurocy, Precision \{km\}

All Stats: 3694 / $-3.794 / 5.650$

loe 5tats: $1017 /-0.564 / 2.352$

Woter Stots: $861 /-7.610 / 0.019$

Phoan P.O.D.: 0,95 Mank P.O.D. =0,82

Act, Cid froe: 0.66 Pasa. Cla Froes 0.53

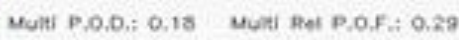

(d)

Figure 10. CALIPSO (Cloud-Aerosol Lidar and Infrared Pathfinder Satellite Observation) data for 21 July 2016 at 0530-0550 UTC, used in Experiment 3: (a) Ground track over a map of the West Pacific Ocean, (b) Ground track over the corresponding Himawari-8 RGB image, (c) Ground track over the corresponding Himawari-8 derived Cloud top height, (d) Statistics for the CALIPSO data.

All previous analysis of variables, validation against radiosonde wind data, and NWP analysis wind data, and best-fit analysis done for Experiment 2 are not repeated here, as both experiments are, as said, equivalent.

Figure 10 depicts the portion of the orbit from Western Australia to Mongolia (red line) for which the CALIPSO data for 21 July 2016 0530-0550 UTC are used in Experiment 3. As seen in Figure 10b, clouds are primarily in the tropics and the Northern part of the pass. On the other hand, over Australia and adjacent ocean to the North, there are few or no clouds, resulting in a limited number of AMVs for comparison with CALIPSO.

Figure 11 depicts, for the different centers, the clouds as detected by CALIPSO (color-coded from gray to red by emissivity).

The tropopause height is designated by the black line across the top of the graph (near the top of the cirrus clouds). The topography of the Earth's surface is the light blue line in the lower part of the graph. In each one of the panels, the collocated AMVs for each center are shown as black asterisk symbols, primarily located near the base of the cirrus cloud feature in the middle of the graph, and at the top of the low-level and medium-level clouds located in the central and right side of the graph.

Overall, the results are in general agreement with the best-fit analysis in Experiment 2; in general, the high-level AMVs need to be adjusted higher in the atmosphere.

AMV heights for the different centers are in good agreement in this specific example, and in apparent disagreement with the previous AMV-pressure scatter plots. However, this is a very small sample, and no attempt has been made to ensure that the AMVs are collocated with each other: data from each center are plotted independently from the other ones. 


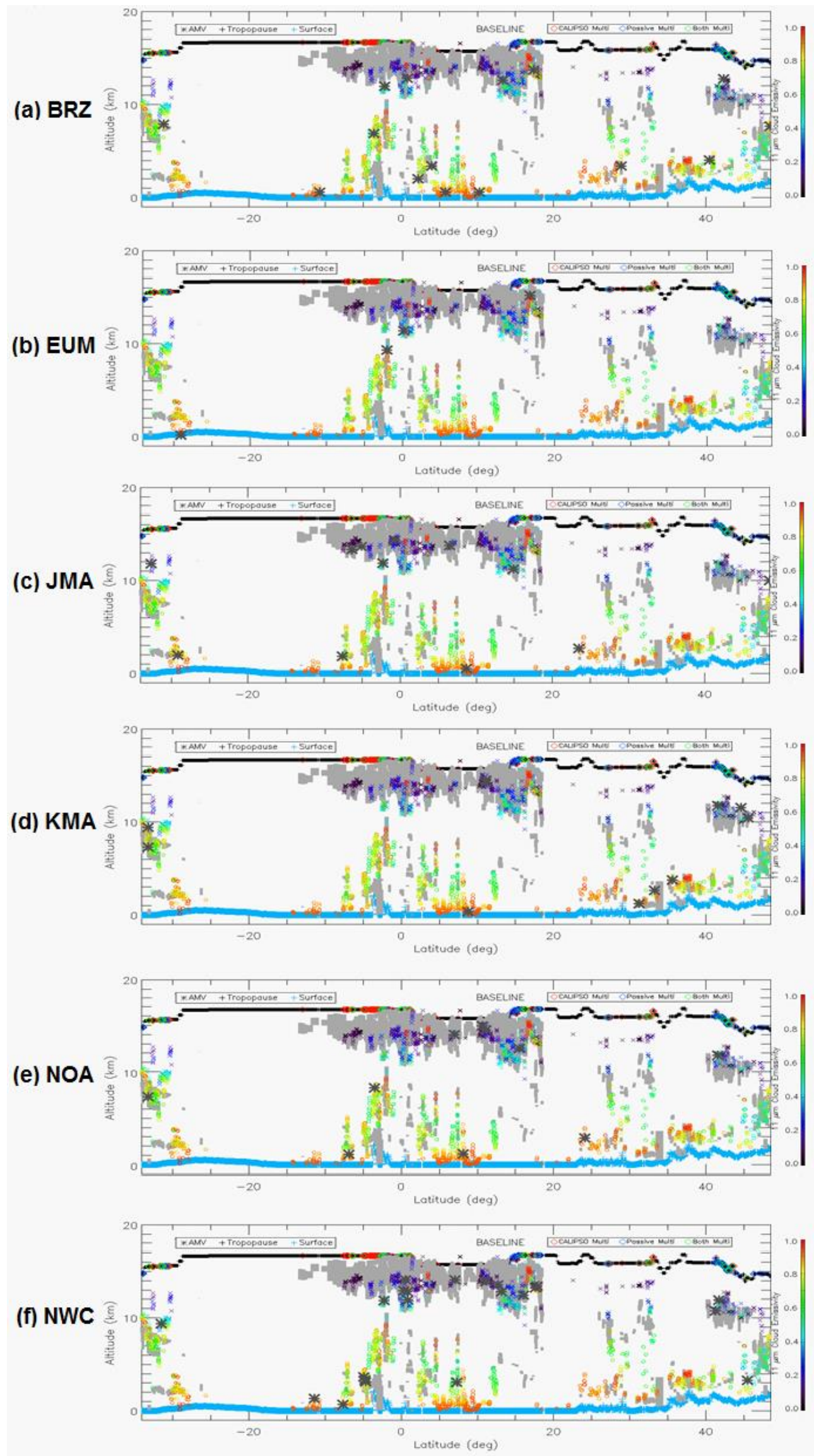

Figure 11. Experiment 3: Collocation of AMVs (defined as black asterisks ${ }^{*}$ ) with CALIPSO cloud measurements for (a) BRZ, (b) EUM, (c) JMA, (d) KMA, (e) NOA, (f) NWC. 


\subsection{Similarities in the AMV Datasets}

To quantify the differences observed in the AMV datasets in Experiments 1 and 2, a 'paired t-test' is used with all combinations of AMV datasets and four considered parameters (speed, direction, pressure, and quality indicator), to determine if the differences in the parameters are statistically significant for collocated pairs of AMVs.

This is done because there is no 'ground truth' for AMVs, and one of the goals of this study is to determine the similarity in the AMVs from the different centers. The statistics are computed using the 'MATLAB paired t-test' function, which performs a ' $t$-test' of the hypothesis that the data come from a distribution with mean zero. The data used here are the differences in each parameter from each pair of AMV datasets; therefore, a mean of zero is expected.

AMVs used with this t-test are quality controlled, retaining only those with QINF $>=50 \%$ and QINF $>=80 \%$, and also those with CQI $>=50 \%$ and CQI $>=80 \%$. For collocation, the distance used here between AMVs is up to $35 \mathrm{~km}$.

Tables 18 and 19 show, respectively, for Experiment 1 (with prescribed configuration) and Experiment 2 (with specific configuration), the pairs of combinations with parameter differences not statistically significant. A total of 15 combinations is defined per parameter (considering all combinations of AMV datasets), and so a total of 60 combinations is defined in each whole test.

Table 18. Similarities found in the AMV datasets by the 'paired t-test' for the different combinations of variables and AMV datasets, in Experiment 1 (with prescribed configuration).

\begin{tabular}{cccccc}
\hline Prescribed Configuration & Speed & Direction & Pressure & Quality Indicator & ALL \\
\hline QINF $>=50 \%$ & 4 & 6 & 1 & 0 & 11 \\
QINF $>=80 \%$ & 6 & 5 & 1 & 1 & 13 \\
CQI $>=50 \%$ & 1 & 3 & 0 & 1 & 5 \\
CQI $>=80 \%$ & 2 & 6 & 1 & 1 & 10 \\
\hline
\end{tabular}

Table 19. Similarities found in the AMV datasets by the 'paired t-test' for the different combinations of variables and AMV datasets, in Experiment 2 (with specific configuration).

\begin{tabular}{cccccc}
\hline Specific Configuration & Speed & Direction & Pressure & Quality Indicator & ALL \\
\hline QINF $>=50 \%$ & 1 & 2 & 0 & 0 & 3 \\
QINF $>=80 \%$ & 1 & 2 & 2 & 0 & 5 \\
CQI $>=50 \%$ & 1 & 2 & 0 & 0 & 3 \\
CQI $>=80 \%$ & 1 & 1 & 0 & 1 & 3 \\
\hline
\end{tabular}

The most consistent agreement is for the AMV direction with the prescribed configuration and the high-quality indicator thresholds. The similarities then reduce progressively for the AMV speed, the AMV quality indicator and the AMV pressure. The lack of agreement in the AMV pressure is likely due to the lack of commonality in the AMV height assignment algorithms; here, though both EUM and NWC centers use 'CCC method' for the height assignment, there are still significant differences for this variable even for these centers.

In general, more homogeneity between variables in different datasets is seen using the same prescribed configuration, using a higher threshold for the 'quality indicator' in the AMV filterings, and using the specific QINF calculated by each center.

This last result seems to be in contradiction with elements shown in the previous sections, in which the CQI provided more homogeneity in the AMV statistics of different centers (comparing, for example, Tables 5 and 6).

The result here could be, however, derived from the fact that with the CQI, the AMV samples of data for all centers are larger, and with this, the 'paired t-test' has more difficulties to show a mean of zero. 


\section{Discussion}

The following are general observations and recommendations from this 'AMV Intercomparison Study':

(1) Experiments 1 and 2 only differ in terms of a prescribed versus specific configuration. Overall, the results between the two experiments are nearly the same in terms of bulk statistics, collocated statistics, comparisons to radiosonde winds and NWP analysis winds, and best-fit analysis, with the main variations related to the number of calculated AMVs. This implies that the impact of using a prescribed versus a specific configuration is small.

(2) For the experiments, the centers have used the 'AMV height assignment' method of their choice. This height assignment method has the biggest impact on the lack of agreement in the AMVs.

(3) The 'Common Quality Indicator (CQI)' has been introduced in this Intercomparison, and it shows some skill in filtering collocated AMVs, resulting in an improved statistical agreement.

(4) Another first for this study has been the addition of CALIPSO data to provide an independent measurement of cloud height, to be used for comparison and validation of the AMV heights. Due to the small sample of collocated observations for the comparison, only a qualitative assessment could be made: AMV heights are generally near the cloud base for the high-level thin cirrus clouds, and near the cloud top for the low-level and the medium-level clouds detected by CALIPSO. For the next round of AMV Intercomparison studies, a comparison of AMVs with MISR winds is planned for additional checkings of the AMVs and the AMV heights.

(5) Even though there is a large variation in the AMV heights for collocated winds, the best-fit analysis and CALIPSO comparison both show that there are equivalent, high-quality winds produced by all centers. This might indicate that the quality control post-processing by each center is an important step in filtering out poor quality AMVs, resulting in a higher quality product. For future Intercomparison studies, an experiment to substantiate this conjecture should be considered.

\section{Conclusions}

The following sections detail the findings from the experiments, in terms of each AMV producer independently. This includes the strengths and weaknesses, as determined by the results of the experiments.

\section{(a) BRZ-Brazil Center for Weather Prediction and Climate Studies (CPTEC/INPE)}

The performance of the BRZ algorithm has improved with respect to the results in the previous AMV Intercomparison. Results are more in agreement with the rest of AMV centers, and differences in many of the variables have reduced. In any case, it is clear that there still exists room for improvement. On the one hand, there are still large differences in the AMV height assignment, as compared to all other centers. This results in BRZ often having the largest deviations from radiosonde winds and NWP analysis winds. On the other hand, BRZ has still to verify the differences found in the direction histograms, in which some directions are found to be much more frequent than other ones.

\section{(b) KMA-Korea Meteorological Administration}

The KMA algorithm performs similarly to the results from the previous AMV Intercomparison. Overall, the comparisons to radiosonde winds and NWP analysis winds are in the middle of the distributions.

\section{(c) EUM-European Organization for the Exploitation of Meteorological Satellites (EUMETSAT)}

The behavior of EUM algorithm is much better when the 'quality indicator threshold' is high $(80 \%)$, and when its specific configuration is used (comparing with the results with the prescribed configuration). In these circumstances, the performance of EUM AMVs is basically similar to that of 
other centers (NOA or NWC). The similarities in the height assignment of EUM and NWC centers are also to be noticed, due to the fact of both using the same 'CCC method' height assignment.

\section{(d) NOA-United States National Oceanic and Atmospheric Administration (NOAA)}

Results for the NOA algorithm have much improved with respect to the previous Intercomparison study, and now their validation results against radiosonde winds and NWP analysis winds are in second position (together with NWC). In any case, there are still some issues with the NOAA algorithm, for example, the vertical distribution of the AMVs, for which there are very few AMVs in middle levels, which is in disagreement with the rest of the centers.

\section{(e) NWC-Satellite Application Facility on support to Nowcasting (NWCSAF)}

NWC algorithm agrees well with other centers in terms of parameter distributions, especially when using the 'common quality indicator' and its specific configuration. Its validation results against radiosonde winds and NWP analysis winds are in second position (together with NOA).

The NWC algorithm is basically the same as the one used for the previous AMV Intercomparison. Due to this stability, its overall performance is similar to what was found in that previous AMV Intercomparison study. However, some elements have been detected that need some analysis by NWC AMV producers: the fact that some AMV directions (e.g., $90^{\circ}$ ) are more frequent in the direction histogram than others in their vicinity.

\section{(f) JMA-Japan Meteorological Agency}

Among the six AMV operational algorithms verified by this AMV Intercomparison, the JMA algorithm is the one with the best performance, considering, in general, all validation and checking elements. This reflects the most important change in all these AMV algorithms when compared to the previous AMV Intercomparison. Specifically, they have the best overall statistics when compared to radiosonde winds and NWP analysis winds, and their best-fit analysis shows that the differences with respect to the best-fit level for JMA AMVs are much smaller than for other centers.

Taking into account the differences in JMA AMV algorithm between the previous AMV Intercomparison study in 2014 [15] and the current one, two main elements have changed: the 'tracking' (for which a 'noise reduction method based on cross-correlation and the averaging of multiple correlation surfaces' is used now) and the 'height assignment' (for which an 'optimal estimation method considering the observed infrared radiances, the vertical profile of the NWP wind, and the estimated brightness temperature using a radiative transfer model' is used now).

The first element affects the quantity and density of AMVs but does not significantly contribute to the improvement in the statistical quality of the AMVs. The second element contributes to the improvement in the statistical quality of the AMVs. Considering that in the previous AMV Intercomparison the performance of JMA was in an intermediate position between all AMV algorithms, the updated JMA height assignment has triggered it to be the best AMV algorithm. However, it is still to be studied if the small differences between the JMA AMVs and the NWP model used for the processing, imply a good impact in later applications like NWP assimilation.

Other processes have evolved throughout the years for the calculation of winds utilizing satellite data as input, including: sea surface winds from satellite scatterometer data, winds with geometric stereoscopic height assignment from multi-angle imagery, wind profiles using optical flow technique from satellite sounder data, wind profiles using the Doppler wind Lidar principle. The next Intercomparisons organized in the two upcoming years should be a good opportunity to compare these techniques against the actual AMV methods used operationally around the world.

Supplementary Materials: The following file is available online at http://www.mdpi.com/2072-4292/11/19/2240/s1: File S1, a zipped file including the following four elements:

- $\quad$ amv_dataset.tar.gz: gzipped tar file with all AMV datasets used in the AMV Intercomparison.

- $\quad$ amv_scripts.tar: tar file with all bash, MATLAB, Python scripts used in the AMV Intercomparison.

- raob.tar.gz: gzipped tar file with the radiosonde data used in the AMV Intercomparison. 
- README_Intercomparison.txt: text file describing and explaining how to process the different 'Supplementary Materials', and how to obtain the 'ECMWF ERA-INTERIM reanalysis' used in the AMV Intercomparison.

Author Contributions: Conceptualization: R.B. and S.W.; Supervision and funding acquisition: J.G.-P.; Resources: R.G.N., R.B., M.C., K.N., K.S., S.M.O., B.-I.L., S.-R.C., J.D., W.B. and J.G.-P.; Methodology, software, formal analysis, and investigation: D.S., I.G., R.D., S.N. and S.W.; Writing-Original draft: D.S. and J.G.-P.; Writing-Review and editing: D.S. and J.G.-P.

Funding: The "Space Science and Engineering Center" (SSEC) of the "University of Wisconsin-Madison" (UW) was funded to do this research by the "European Organization for the Exploitation of Meteorological Satellites (EUMETSAT)", through the "Satellite Application Facility on Support to Nowcasting and Very short range forecasting (NWCSAF)" “Visiting Scientist Activity (VSA)" program.

Acknowledgments: This research has been considered from the general interest by the "International Winds Working Group (IWWG)", to monitor and intercompare the different geostationary AMV algorithms available in the world in a common situation. The IWWG wants to thank colleagues from the "Space Science and Engineering Center" (SSEC) of "University of Wisconsin-Madison" (UW), for the work done with a very tight schedule. In addition, the IWWG thanks:

- $\quad$ The colleagues in the different AMV production centers for the effort to provide the AMV datasets for this AMV Intercomparison: Renato Galante Negri (BRZ), Régis Borde and Manuel Carranza (EUM), Kenichi Nonaka and Kazuki Shimoji (JMA), Soo Min Oh, Byung-Il Lee and Sung-Rae Chung (KMA), Jaime Daniels and Wayne Bresky (NOA) and Javier García-Pereda (NWC).

- $\quad$ Régis Borde and Manuel Carranza (EUM), Kenichi Nonaka and Kazuki Shimoji (JMA), and Wayne Bresky (NOA) for the definition and preparation of the input data for the calculation of the AMVs in the different centers.

Conflicts of Interest: The whole formal analysis and the investigation were made by researchers completely independent of all AMV algorithms involved in the research. However, some of the AMV producers participated in the definition and preparation of the common input data for the calculation of the AMVs (Régis Borde, Manuel Carranza, Kenichi Nonaka, Kazuki Shimoji, and Wayne Bresky), and the supervision and writing of the manuscript (Javier García-Pereda). The IWWG and all AMV producers were aware of this and in agreement with these circumstances.

\section{References}

1. Menzel, W.P. Cloud Tracking with Satellite Imagery: From the Pioneering Work of Ted Fujita to the Present. Bull. Am. Meteorol. Soc. 2001, 82, 33-47. [CrossRef]

2. Novak, C.; Young, M. The operational processing of wind estimates from cloud motions: Past, present and future. In Proceedings of the 11th International Symposium on Remote Sensing Of Environment: Application And Processing of Remotely Sensed Data, Ann Arbor, MI, USA, 25-29 April 1977.

3. Bresky, W.C.; Daniels, J.M.; Bailey, A.A.; Wanzong, S.T. New methods towards minimizing the slow speed bias associated with atmospheric motion vectors. J. Appl. Meteorol. Climatol. 2012, 51, 2137-2151. [CrossRef]

4. Smith, E.; Phillips, D. WINDCO: An Interactive System for Obtaining Accurate Cloud Motions from Geostationary Satellite Spin Scan Camera Pictures: Measurements from Satellite Platforms; Scientific Rep. AAS5-115042; Space Science and Engineering Center, University of Wisconsin-Madison: Madison, WI, USA, 1972; pp. 1-51.

5. Nieman, S.; Smith, W.L.; Hayden, C.M.; Gray, D.; Wanzong, S.T.; Velden, C.S.; Daniels, J. Fully automated cloud-drift winds in NESDIS operations. Bull. Am. Meteorol. Soc. 1997, 78, 1121-1133. [CrossRef]

6. Schmetz, J.; Holmlund, K.; Hoffman, J.; Strauss, B.; Mason, B.; Gaertner, V.; Kock, A.; DeBerg, L.V. Operational cloud-motion winds from Meteosat infrared images. J. Appl. Meteorol. 1993, 32, 1206-1225. [CrossRef]

7. Borde, R.; Doutriaux-Boucher, M.; Dew, G.; Carranza, M. A Direct Link between Feature Tracking and Height Assignment of Operational EUMETSAT Atmospheric Motion Vectors. J. Atmos. Ocean. Technol. 2014, 31, 33-46. [CrossRef]

8. Kodaira, N.; Kato, K.; Hamada, T. Man-machine Interactive Processing of Extracting Cloud Top Height and Cloud Wind Data from GMS Images. Meteorol. Satell. Center Tech. Note 1979, 1, 59-78.

9. Machado, L.A.T.; Ceballos, J. Satellite Based Products for Monitoring Weather in South America: Winds and Trajectories. In Proceedings of the 5th International Winds Workshop, Saanenmöser, Switzerland, 20-23 October 1998.

10. Negri, R.G.; Machado, L.A.T. CPTEC/INPE Operational GOES-10 Atmospheric Motion Vectors. In Proceedings of the 9th International Winds Workshop, Annapolis, MD, USA, 14-18 April 2008. 
11. National Institute of Meteorological Sciences (NIMS). Development of Meteorological Data Processing System for Communication, Ocean and Meteorological Satellite; National Digital Science Library (NDSL): Seoul, Korea, 2009; vol. 1, pp. 77-101. Available online: http://www.ndsl.kr/ndsl/commons/util/ndslOriginalView.do?dbt= TRKO\&cn=TRKO201000010174 (accessed on 24 September 2019).

12. García-Pereda, J.; Fernández-Serdán, J.M.; Alonso, Ó.; Sanz, A.; Guerra, R.; Ariza, C.; Santos, I.; Fernández, L. NWCSAF High Resolution Winds (NWC/GEO-HRW) Stand-Alone Software for Calculation of Atmospheric Motion Vectors and Trajectories. Remote Sens. 2019, 11, 2032. [CrossRef]

13. Genkova, I.; Borde, R.; Schmetz, J.; Daniels, J.; Velden, C.; Holmlund, K. Global atmospheric motion vector intercomparison study. In Proceedings of the 9th International Winds Workshop, Annapolis, MD, USA, 14-18 April 2008.

14. Genkova, I.; Borde, R.; Schmetz, J.; Velden, C.; Holmlund, K.; Bormann, N.; Bauer, P. Global atmospheric motion vector intercomparison study. In Proceedings of the 10th International Winds Workshop, Tokyo, Japan, 22-26 February 2010.

15. Santek, D.; Velden, C.; Genkova, I.; Wanzong, S.; Stettner, D.; Mindock, M.; García-Pereda, J. 2014 AMV Intercomparison Study. In Proceedings of the 12th International Winds Workshop, Copenhagen, Denmark, 16-20 June 2014.

16. Negri, R.G.; Borde, R.; Carranza, M.; Nonaka, K.; Oh, S.M.; Bailey, A.; Wanzong, S.; García-Pereda, J. International Winds Working Group (IWWG) Operational AMV Product Survey. Available online: http: //cimss.ssec.wisc.edu/iwwg/Docs/AMVSURVEY2018_TOTAL.pdf (accessed on 24 September 2019).

17. Holmlund, K. The utilisation of statistical properties of satellite derived Atmospheric Motion Vectors to derive Quality Indicators. Weather Forecast. 1998, 13, 1093-1104. [CrossRef]

18. Dee, D.P.; Uppala, S.M.; Simmons, A.J.; Berrisford, P.; Poli, P.; Kobayashi, S.; Andrae, U.; Balmaseda, M.A.; Balsamo, G.; Bauer, P.; et al. The ERA-Interim reanalysis: Configuration and performance of the data assimilation system. Q. J. R. Meteorol. Soc. 2011, 137, 553-597. [CrossRef]

19. Salonen, K.; Cotton, J.; Bormann, N.; Forsythe, M. Characterizing AMV height-assignment error by comparing best-fit pressure statistics from the Met Office and ECMWF data assimilation systems. J. Appl. Meteorol. Climatol. 2015, 54, 225-242. [CrossRef]

(C) 2019 by the authors. Licensee MDPI, Basel, Switzerland. This article is an open access article distributed under the terms and conditions of the Creative Commons Attribution (CC BY) license (http://creativecommons.org/licenses/by/4.0/). 